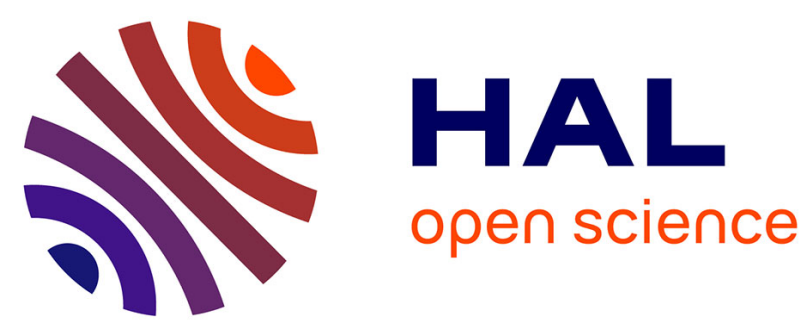

\title{
Impact of Short-Time Fourier Transform Parameters on the Accuracy of EMI Spectra Estimates in the 2-150 $\mathrm{kHz}$ Supraharmonic Interval
}

Leonardo Sandrolini, Andrea Mariscotti

\section{> To cite this version:}

Leonardo Sandrolini, Andrea Mariscotti. Impact of Short-Time Fourier Transform Parameters on the Accuracy of EMI Spectra Estimates in the $2-150 \mathrm{kHz}$ Supraharmonic Interval. Electric Power Systems Research, 2021, 195, pp.107130. 10.1016/j.epsr.2021.107130 . hal-03248136

\section{HAL Id: hal-03248136 \\ https://hal.science/hal-03248136}

Submitted on 3 Jun 2021

HAL is a multi-disciplinary open access archive for the deposit and dissemination of scientific research documents, whether they are published or not. The documents may come from teaching and research institutions in France or abroad, or from public or private research centers.
L'archive ouverte pluridisciplinaire HAL, est destinée au dépôt et à la diffusion de documents scientifiques de niveau recherche, publiés ou non, émanant des établissements d'enseignement et de recherche français ou étrangers, des laboratoires publics ou privés. 


\title{
Impact of Short-Time Fourier Transform Parameters on the Accuracy of EMI Spectra Estimates in the 2-150 $\mathrm{kHz}$ Supraharmonic Interval
}

\author{
Leonardo Sandrolini ${ }^{\mathrm{a}, *}$, Andrea Mariscotti ${ }^{\mathrm{b}}$ \\ ${ }^{a}$ Department of Electrical, Electronic, and Information Engineering "Guglielmo Marconi," \\ University of Bologna, I-40136 Bologna, Italy \\ ${ }^{b}$ Department of Electrical, Electronic and Telecommunications Engineering and Maritime \\ Architecture, University of Genova, Genova, Italy
}

\begin{abstract}
Methods based on time-domain sampling and successive tapering and Fourier analysis, used in the "harmonics" frequency interval up to $2.4 \mathrm{kHz}$, cannot be automatically extended to the $2-150 \mathrm{kHz}$ interval, in particular considering the emissions of Switched-Mode Power Supplies (SMPSs) in a modern smart grid or low-voltage distribution scenario. Emissions are substantially impulsive and oscillatory superposed to the mains signal, possibly featuring multiple switching fundamentals and their harmonics with unstable or purposely changing fundamental frequency. Fourier analysis (namely Discrete Fourier Transform) is commonly used to characterize the spectrum of emissions from time-domain data, but the influence of its settings should be verified for accuracy and systematic errors, so to establish the uncertainty of spectral estimates. After an initial theoretical and practical discussion of characteristics, objectives and weak points, a verification is carried out with the help of several measurements performed in different operating conditions on a set of six different SMPSs, evaluating systematic error and uncertainty, and defining a set of recommendations.
\end{abstract}

Keywords: AC-DC power converters; Discrete Fourier Transform;

Electromagnetic compatibility; Electromagnetic interference; Conducted

\footnotetext{
* Corresponding author

Email addresses: leonardo.sandrolini@unibo.it (Leonardo Sandrolini), andrea.mariscotti@unige.it (Andrea Mariscotti)
} 
emissions; Power Quality; Smart grids; Supraharmonics.

\section{Introduction}

The widespread use of Switched-Mode Power Supplies (SMPSs) can be justified with flexibility, portability and better efficiency, including superior voltage regulation at the load compared to more traditional conversion schemes. SMPSs feature various types of power electronic converters, whose conducted emissions must be assessed accurately, with harmonic and interharmonic distortion occurring over a wide range of frequencies [1. Conducted emissions caused by the switching process, in fact, originate from switching fundamentals of some tens of $\mathrm{kHz}$ and spread in the hundreds $\mathrm{kHz}$ and $\mathrm{MHz}$ ranges, well above the "classical" interval of harmonic penetration studies, limited in general to $2.0-2.4 \mathrm{kHz}$ for 50-60 Hz systems 2, 3. In the last ten years attention has been extended to frequency intervals up to $150 \mathrm{kHz}$ [4] 12 , focusing on the so called "supraharmonics". Some standards [13 16] deal with the $2-150 \mathrm{kHz}$ interval, but do not cover explicitly a critical source of emissions, SMPSs, and similarly other switching power converters, such as Photovoltaic Infeed Converters (PVIC). Modern SMPS as a source of EMI in smart grids and LV industrial networks have received much attention $4-12$, especially considering the extension of the frequency range beyond the harmonic interval and the significant propagation and penetration in low-voltage networks (e.g. caused by interaction of device frontend filters, resonances, etc.) [17 19]. A broader resolution bandwidth has been recently proposed [7, 20, passing from the aggregation bandwidth of $200 \mathrm{~Hz}$ of the IEC 61000-4-7 [21], to the $2 \mathrm{kHz}$ frequency resolution of the IEC 61000-4-30 [20, closer to the resolution bandwidth (RBW) required for the radiofrequency conducted emissions above $150 \mathrm{kHz}$. All these measurements are performed using timesampled data followed by spectrum calculation with Fourier transform (a Discrete Fourier Transform, DFT, implemented as Short-Time Fourier Transform, STFT, to include the overlap of adjacent windows when tracking Fourier spectrum versus time). When dealing with potentially nonstationary 
impulsive emissions, the effect of the STFT parameters on the accuracy of the spectrum estimate in amplitude and frequency needs to be quantified. Nevertheless, frequency domain scan of emissions with classic EMI receivers requires measuring the signal within the resolution bandwidth in a given measurement time, which increases the scan time even for narrow frequency ranges such as $2-150 \mathrm{kHz}$, as the receiver must be tuned in steps smaller than the resolution bandwidth (less than $200 \mathrm{~Hz}$, the $6 \mathrm{~dB}$ EMI bandwidth according to [22]) to obtain an accurate result [23, 24]. Although the cited standards require the evaluation of amplitude-only spectra against limits, the phase of the spectrum components may be also relevant to evaluate the combined effects of multiple SMPSs, resonance phenomena, etc. [25], giving advantage to methods based on time-domain data.

In general, SMPS conducted emissions observed in time domain contain the switching fundamental (or more than one, depending on the architecture), its harmonics, and other narrowband components at higher frequency, superposed to the mains fundamental and repeating following the modulation process (e.g. $100 \mathrm{~Hz}$ for power factor correction at the output of the single-phase rectifier with $50 \mathrm{~Hz}$ mains), as shown in Fig. 2 of [26] and later in sec. 3 below.

Considering the switching and modulation process, modern modulators for converters in the range of some tens of $\mathrm{W}$ up to some $\mathrm{kW}$ have switching frequencies in the tenths of $\mathrm{kHz}$ and adopt smart modulation techniques to reduce voltage stress on components, improve efficiency and reduce EMI. Modulators are also able to switch between different modulation patterns, each optimized for a range of operations: for example pulse skipping at very low loads improves efficiency, whereas Pulse Frequency Modulation (PFM) not only does not sacrifice efficiency, but has a very fast dynamic response, particularly useful for load steps. The well known Pulse Width Modulation (PWM) is usually adopted at large loading near the nominal or above, to ensure stability, when attaining high values of efficiency is much less critical. These modulation techniques have different spectral signatures, and considering the fast control loop dynamics, they may be applied one after the other during the same transient or load step, with 
two of them even captured in a single Fourier time window, that needs thus to be as short as possible.

In addition, small-scale variability of spectral lines and phase noise can also be caused by drift due to temperature changes, switching timebase instability, etc., besides small modulation adjustments.

As opposed to the standard swept frequency-domain method using a spectrum analyzer or EMI receiver with Peak, Quasi-Peak or Average detectors as per many standards, real-time spectrum analyzers have been proposed and demonstrated [27 29], as well as pure time-domain baseband sampling, e.g. by means of a Digital Sampling Oscilloscope (DSO) or data acquisition system (DAS) followed by Fourier analysis, is used extensively [30, 31. The advantages are: economic, if using a DSO or DAS already available; the possibility of selecting various analysis modes and parameters, and repeating the analysis at will starting from the original time-sampled data; tracking frequency domain characteristics of emissions for the entire spectrum, with the zero span mode limited to a single frequency only. Conversely, the drawbacks and limitations are: a more limited dynamic range and higher noise floor, as a direct consequence of the limited number of bits; the risk of overloading, that may occur whenever large low-frequency components or major transients overlap to the frequency interval subject to measurement. Overloading from low-frequency components can be prevented by using a high-pass filter (similar to the EMI receiver input selector filter), but cannot improve the full scale setting, which is determined by the height of the main peak, as it will be shown in Section 3 .

This work considers time-domain sampling, but follows a frequency-domain approach based on Fourier analysis, as the basic analysis tool so widespread in the literature, for which some care must be exercised. In fact, Fourier analysis is implemented in the methods described in the two major referenced standards for supraharmonics, IEC 61000-4-7 [21] and IEC 61000-4-30 [20]. Recently in [32, 33. these two approaches have been compared and, although both methods present critical elements in detecting components with variable frequency and/or magnitude, IEC 61000-4-30 shows better performance than IEC 61000- 
4-7, except for the analysis of disturbance components with small amplitude. A method that removes spacing between adjacent Fourier windows allows highfrequency components to be detected in a periodic voltage disturbance appearing as a notch, which instead cannot be detected by equally-spaced measurement methods, such as the one in [20]. This method in reality corresponds to the application of STFT for the analysis of long sequences of time-sampled data; the correct and aware use of the STFT to this wide class of signals needs thus an in-depth investigation, as it will be shown in the rest of the work.

It is briefly noted that although the Fourier analysis is the preferred and widely used approach, analysis of electrical quantities with poor stationarity or featuring significant transients can be carried out using time-domain Power Quality indexes and Multiresolution Signal Decomposition has been proposed with examples for AC and DC supply schemes [34 36].

The objective of the paper is twofold: i) defining suitable choices of STFT parameters as for frequency resolution, sampling rate, overlap and time window to track satisfactorily signal dynamics, including agreement with existing standards; ii) characterizing the impact of the various parameters on the accuracy of the estimate of spectral components (amplitude and frequency). Considering the lack of synchronous sampling and local non-stationarity of the SMPS modulation, spectral leakage is an issue: a relevant point is thus to include the effect of smoothing windows in the assessment.

The characteristics of the STFT algorithm considered to process time-domain data and the experimental setup are illustrated in Sec. 2. The results are presented in Sec. 3. From the analysis presented in Sec. 4, it is shown that the choice of the smoothing window has influence on the accuracy of the amplitude estimate and represents a source of systematic error. Although broader smoothing windows tend to worsen the accuracy of the frequency estimate of components, this is not a critical aspect, as interpolation can recover easily the location of signal components lying between frequency bins with a more than satisfactory accuracy. Finally, the estimates are statistically analysed by means of boxplots, where the impact of different windows, overlap and resolu- 
tion frequency on the estimated amplitude of spectral peaks is considered. A set of recommendations is defined as regards max-hold setting, type of window, overlap and frequency resolution.

The analysis is verified against experimental data from six different SMPS in various operating conditions, so with a wide range of spectral signatures. Swept frequency spectra of an EMI receiver are also included as reference.

\section{Problem Definition and Experimental Setup}

The considered characteristics of the STFT algorithm are reviewed in the following, setting the framework of the analysis appearing in Sections III and IV. The experimental setup for the measurement of SMPS conducted emissions is also described.

\subsection{STFT General Characteristics}

The STFT is based on the Discrete Fourier Transform (DFT) and adopts a sliding approach to track spectral properties of the signal over time. The critical parameters are: the time window $T$, which defines the frequency resolution, $d f=1 / T$, and sets a relationship with the signal nonstationary components; the number of points $N$ stems directly from the chosen sampling frequency $f_{s}=N d f$. It is worth noting that DFT can be interpreted as a bank of filters each with $d f$ bandwidth, useful when reasoning on the amount of noise and averaging of nonstationary components implemented by a more or less large $d f$ value.

Due to nonstationarity and lack of synchronous sampling, spectral leakage [37, 38] is a key performance issue in both its manifestations:

- Long-range leakage: the interference, or mutual effect, between spectral components, caused by the lack of synchronism between the time window $T$ and the period of the sinusoidal components within the signal. When synchronous sampling is not possible, a suitable control measure is using smoothing (or tapering) windows to reduce the order of the discontinuity at the ends of the periodic extension where the signal is cut. 
- Short-range leakage, that corresponds to the simpler fact that, when the spectrum is represented as samples on a discrete frequency vector, the real underlying signal frequency may lie between two discrete points, thus worsening or biasing the frequency estimate; it is also named "scalloping loss" or "picket fence effect". The problem is remedied by interpolation.

Smoothing windows have impact on many attributes of spectral analysis, including detection of details, resolution, and dynamic range. The estimated harmonic amplitude at a given frequency is biased by the accumulated broad-band noise that falls inside the bandwidth of the window, behaving as a filter. Starting from the implicit rectangular window, a convenient figure of merit is the Equivalent Noise Bandwidth (ENBW) [37] (see Table I). To ensure spectrum readability, the dynamic range between the relevant components peaks and two types of extraneous elements (noise and residual leakage due to window sidelobes) should be considered. For noise, without synchronous sampling, the improvement of such dynamic range by averaging of $N_{s}$ successive spectra is only through the reduction of the noise variance (as square root of $N_{s}$ ), that is the so-called "incoherent averaging" using only amplitude information [38. For sidelobes a window with low "highest sidelobe level" should be used 37.

Interpolation is a further option to improve the accuracy of amplitude and frequency estimates: several techniques have been proposed [39 42], such as parabolic, Gaussian (for samples with logarithmic amplitude, such as spectra expressed in $\mathrm{dB}$ ), and $M$-point interpolation ( $M$ odd), with discussion still open on accuracy and applicability with/without smoothing windows.

STFT introduces another parameter, the percentage of overlap $p$ between adjacent time windows for better time tracking, with a minor, but not negligible, impact on the correlation between spectral components.

\subsection{STFT Settings}

The values of the parameters for the analysis have been scheduled with different exigencies: 
- for the frequency resolution $d f$ and time window length $T$ :

- compliance with the IEC 61000-4-7 21] requirement for PQ analysis, applicable to the low frequency range up to $2 \mathrm{kHzfor} 50 \mathrm{~Hz}$ systems, setting $T=200 \mathrm{~ms}$; this sets the minimum overall time record length to $200 \mathrm{~ms}$;

- compliance with the standards for EMI in the 2-150 kHz range [1316], that indicate a swept frequency-domain technique using preferably $200 \mathrm{~Hz}$ resolution bandwidth (RBW) (corresponding to $T=$ $5 \mathrm{~ms})$;

- to the aim of extending the $200 \mathrm{~Hz}$ analysis and covering MILSTD settings, a $1 \mathrm{kHz}$ RBW is included and an additional smaller $\mathrm{RBW}=100 \mathrm{~Hz}$ (corresponding to $T=1$ and $10 \mathrm{~ms}$, respectively);

- for the overlap percentage $p$ :

- values used in practice are 0 and $50 \%$ minimizing the correlation between samples of adjacent windows; the $0 \%$ value will be discouraged in section IV;

- selected smoothing windows are a tradeoff between completeness and compactness: the seven most popular windows (von Hann, Hamming, BlackmanHarris, Bohman, Flat Top, Gaussian, and the Rectangular one) are selected out of a set of about 25 available [37. (see Table 1).

\subsection{Experimental Setup}

Six SMPSs for Information Technology Equipment (ITE) are considered (see Table 2), featuring low nominal power (between 10 and $30 \mathrm{~W}$ ), to ease load power sizing (metal-heatsink housed resistors were used) and allow the use of a small 16 A LISN (Line Impedance Stabilization Network).

The AC mains disturbance, as an external factor and a source of measurement error, is decoupled by the LISN [43]. The variations of the fundamental 
Table 1: Properties of the Selected Smoothing Windows

\begin{tabular}{|c|c|c|c|c|c|}
\hline Window & $\begin{array}{c}\text { Highest } \\
\text { Side-lobe } \\
\text { level (dB) }\end{array}$ & $\begin{array}{c}\text { Coherent } \\
\text { Gain }\end{array}$ & $\begin{array}{c}\text { Equiv. } \\
\text { Noise } \\
\text { Band- } \\
\text { Width } \\
\text { (bins) }\end{array}$ & $\begin{array}{c}\text { Scallop } \\
\text { Loss (dB) }\end{array}$ & $\begin{array}{c}\text { Worst-case } \\
\text { processing } \\
\text { loss (dB) }\end{array}$ \\
\hline \hline $\begin{array}{c}\text { Rectangular } \\
(\mathrm{R})\end{array}$ & -13 & 1.000 & 1.00 & 3.92 & 3.92 \\
\hline $\begin{array}{c}\text { von Hann } \\
(\text { HN) }\end{array}$ & -32 & 0.500 & 1.50 & 1.42 & 3.18 \\
\hline $\begin{array}{c}\text { Hamming } \\
(\text { HM) }\end{array}$ & -27 & 0.540 & 1.36 & 1.75 & 3.10 \\
\hline $\begin{array}{c}\text { Blackman- } \\
\text { Harris } \\
(\mathrm{BH})\end{array}$ & -92 & 0.359 & 2.00 & 0.83 & 3.85 \\
\hline Bohman (B) & -46 & 0.405 & 1.79 & 1.02 & 3.54 \\
\hline Flat Top (FT) & -88 & 0.216 & 3.77 & 0.01 & 5.77 \\
\hline Gaussian (G) & -44 & 0.495 & 1.45 & 1.58 & 3.18 \\
\hline
\end{tabular}


Table 2: List of Tested SMPSs and Load Conditions

\begin{tabular}{|c|c|c|c|c|}
\hline Acronym & SMPS & $\mathrm{V}_{\text {no-load }}[\mathrm{V}]$ & $\begin{array}{c}\text { P25 } \mathrm{I}_{25 \% \text {-load }} \\
{[\mathrm{A}]}\end{array}$ & $\begin{array}{c}\text { P90 I }{ }_{90 \%-l o a d} \\
{[\mathrm{~A}]}\end{array}$ \\
\hline LW & $\begin{array}{c}\text { Lacie White } \\
(24 \mathrm{~W})\end{array}$ & 12.94 & 0.51 & 1.83 \\
\hline $\mathrm{LB}$ & $\begin{array}{l}\text { Lacie Black } \\
(26.4 \mathrm{~W})\end{array}$ & 11.75 & 0.47 & 1.72 \\
\hline $\mathrm{KT}$ & KTEC (18W) & 12.19 & 0.37 & 1.43 \\
\hline $\mathrm{SH}$ & $\begin{array}{c}\text { Shenzhen } \\
\text { Honor }(18 \mathrm{~W})\end{array}$ & 12.45 & 0.38 & 1.45 \\
\hline $\mathrm{BA}$ & Balance (10W) & 5.212 & 0.51 & 1.94 \\
\hline KA & Kario $(10 \mathrm{~W})$ & 5.144 & 0.51 & 1.91 \\
\hline
\end{tabular}

amplitude instead are not filtered by the LISN and should be considered as a potentially influencing factor. Recently, they have been considered for their impact on the assessment of conducted emissions of LED lamps [43, finding that the LISN (or equivalent network) is necessary to filter pollution of supraharmonic terms from the mains, but waveform quality for the low frequency portion can be ensured only with a separated power supply. Results obtained in [43, Fig. 12, show two different behaviors for Type A and Type D LED lamps, with the former quite insensitive to a spread of the fundamental amplitude of $\pm 10 \%$; the latter instead exhibit a $100 \%$ variation of current intensity at the switching frequency over the said spread. In the present case, the analysis is carried out for each SMPS and each test condition with one recorded trace of $200 \mathrm{~ms}$, where significant variations of mains amplitude can be excluded a priori. Evaluation of the rms of the fundamental in recorded data confirmed a short-term (200 ms, i.e. within one record) and long-term (1 hour, i.e. between selected records) variability of about $0.25-0.35 \%$ and $0.8-0.9 \%$, respectively, and thus negligible. 
Only one value of the load current is reported for the $25 \%$ and $90 \%$ load conditions, with an approximation of less than $1 \%$ for variability due to self heating during each set of tests.

The SMPS is connected to the AC mains through the LISN to carry out the measurements both in the time- and frequency- domain. Time-domain data are sampled with an 8-bit DSO at a sampling rate of $10 \mathrm{MSa} / \mathrm{s}$, to keep records to a manageable size for later post-processing. An anti-aliasing low-pass filter with a cutoff frequency of $2 \mathrm{MHz}$ is also used: the resistor $R_{2}$ is $5 \mathrm{k} \Omega$ and the capacitance $C_{2}$ is the internal input capacitance of the DSO, equal to $15 \mathrm{pF}$ with good accuracy (and not critical for the used sampling rate compared to the selected $2 \mathrm{kHz}-1 \mathrm{MHz}$ interval). A high-pass filter with a cutoff frequency of $1 \mathrm{kHz}$ (one octave below the minimum frequency of interest) is also included to attenuate the fundamental and the low-order harmonics possibly leaking through the LISN: the resistor $R_{1}=1.5 \mathrm{k} \Omega$ is large enough to be neglected in parallel to the impedance matching $50 \Omega$ resistor $R_{m}$, giving a neat $100 \mathrm{nF}$ for $C_{1}$ to get the $1 \mathrm{kHz}$ cutoff. This setting is also in line with the EN 61000-4-30. For frequency domain measurements an EMI receiver with Peak detector and variable RBW was used, scanning between $10 \mathrm{kHz}$ and $1 \mathrm{MHz}$. The experimental setup is shown in Fig. 1 .

The IEC 61000-4-7 [21] indicates a requirement of synchronization with the mains fundamental waveform and period; the reason is to control spectral leakage at the source, rather than relying entirely on tapering in post-processing during Fourier analysis. As a matter of fact this is relevant for harmonics, i.e. namely spectral components harmonically related to the mains frequency. In the present case observed phenomena are switching phenomena and consequential ringing, that are not harmonics of the fundamental, although they occur as a pulse train synchronized with the double of the fundamental frequency $(100 \mathrm{~Hz})$. This fact is considered then later in sec. 3.1 for the selection of the window duration for the Fourier analysis. The pulsed pattern is thus synchronized with the mains frequency, and as a consequence DSO recording triggered to the main pulse, although the continuous Fourier spectrum of a single pulse is not related 


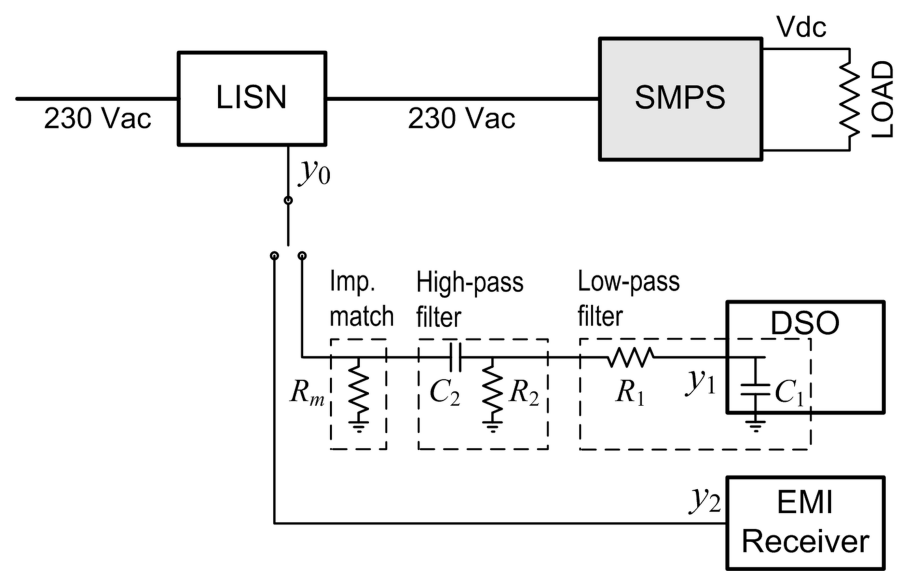

Figure 1: Scheme of the experimental setup: SMPS under test and its dc variable load, LISN (Line Impedance Stabilization Network), $50 \Omega$ impedance matching, high-pass filter (1 kHz cutoff) and low-pass filter (2 MHz cutoff), Digital Storage Oscilloscope (DSO) and EMI Receiver.

to it. Conversely, EMI receiver scans are not synchronized, as commonplace for RF conducted emission measurements, and the duration of the reading of each frequency bin (dwell time) is sufficiently longer than the $100 \mathrm{~Hz}$ period, as explained later.

\section{Results Overview and General Considerations}

This section reports a synthesis of the results and an overview of the achieved accuracy, as a guide for the interpretation of the detailed results shown in the next section.

\subsection{Time domain waveforms}

All SMPS have the signal $y_{1}$ from the LISN to the DSO characterized by impulses repeating every $10 \mathrm{~ms}$ (corresponding to the ripple of the rectified mains voltage internally in the SMPS), with alternate waveshape, depending on the internal alternating polarity of the rectification stage (see Fig. 2). Each impulse contains some bursts of oscillations with variable damping. 
For the window duration $T$ three conditions may be identified: $T<10 \mathrm{~ms}$, $T=10 \mathrm{~ms}$ and $T>10 \mathrm{~ms}$. A long window with $T \gg 10 \mathrm{~ms}$ will bracket many impulses of either polarity. Choosing exactly $10 \mathrm{~ms}$ with bad synchronization, the risk of observing always a head and a tail with opposite polarity (and thus smaller mean value) is quite high. Overlapping these $10 \mathrm{~ms}$ windows will ensure that there will be one window capturing an almost aligned portion of the impulse, much closer to the desired amplitude estimate. A value of $T$ only slightly larger than $10 \mathrm{~ms}$ will be able to capture the impulse whatever the initial time position avoiding the negative effects of "weird" synchronization for $T=10 \mathrm{~ms}$. Using a window conveniently shorter than $10 \mathrm{~ms}$ ensures that bracketing of the central impulses will occur without reduction due to averaging with smaller portions in the head and tail of the signal; in this case the estimate of the overall spectrum will result from the collection and suitable processing of a sufficiently long series of windows. So, this last setting should be the preferred one.

Since the portion of the impulse yielding the peak amplitude is quite narrow (occurring at 10 and $20 \mathrm{~ms}$ in Fig. 2(a), and accompanied by additional impulses in Fig. 2(b)), taking the maximum absolute value of the STFT spectra (but losing phase information) will give a correct and consistent result, provided that $T$ is not too small, e.g. not less than $0.5-1 \mathrm{~ms}$ in the present case. It is observed that $0.5 \mathrm{~ms}$ agrees with the $2 \mathrm{kHz}$ bandwidth requirement in [20].

\subsection{Spectrum characteristics}

The spectrum of the conducted emissions with a waveform as in Fig. 2 is a line spectrum as could be measured with a narrow enough $\mathrm{RBW}$, in the range of $30 \mathrm{~Hz}$ or less, to separate the lines spaced by $100 \mathrm{~Hz}$ (twice the mains frequency). A swept frequency-domain measurement as in Fig. 3 can measure the rms at each step with a dwell time long enough to assure having captured the maximum rms reading of the signal at that frequency bin (the scan time of the example was about 11 hours).

The first peaks of the switching fundamental appear between 20 and $50 \mathrm{kHz}$ depending on the type of SMPS; their harmonics have decreasing amplitude, 


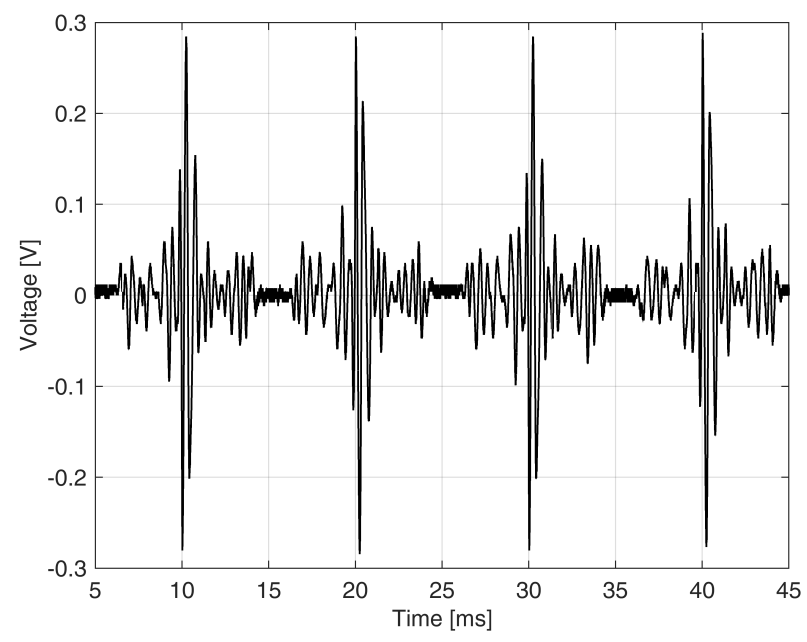

(a)

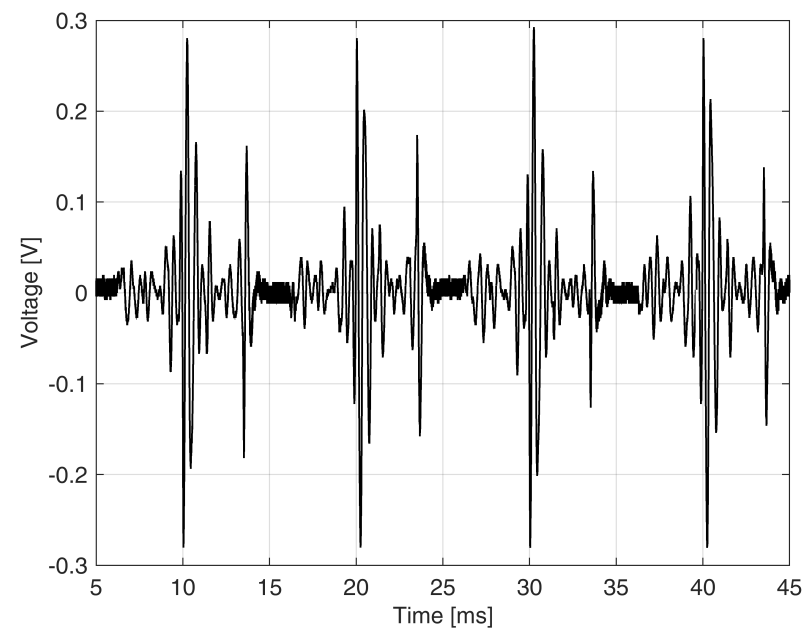

(b)

Figure 2: Time domain waveform of the high-pass filtered LISN output $y_{1}$ for KT P25 (a) and P90 (b) power levels. 
until they are too close to the noise floor to be recognized. Other components of a more or less transient nature can also be recognized.

The spectrum of the KTEC SMPS for the P25 condition (whose waveform is shown in Fig. 2 (a)) is displayed in Fig. 4 for various $T$ values, attempting to capture and display the relevant spectral characteristics. The tradeoff between accurate frequency location and amplitude estimate is clear: the first two curves for 10 and 5 ms identify a main low-frequency component near $2.5 \mathrm{kHz}$, underestimating the amplitude of the peak; the $0.5 \mathrm{~ms}$ window instead gives a peak amplitude of $108.6 \mathrm{~dB} \mu \mathrm{V}$, quite close to the observed peak-to-peak value of $0.564 \mathrm{~V}$, that corresponds to a theoretical maximum value of exactly $109 \mathrm{~dB} \mu \mathrm{V}$. The spectrum then has a slope of about $1 / f^{2}$ to $1 / f^{3}$ down to $20-40 \mathrm{kHz}$, where the profile is approximately flat.

The correspondence between the swept-frequency spectrum of Fig. 3 and the STFT with max hold of Fig. 4(b) can be assessed once it is established the correspondence between the RBW of $10 \mathrm{~Hz}$ and e.g. the $d f=1 \mathrm{kHz}$ of the intermediate curve of Fig. 4. 10 peaks around $2 \mathrm{kHz}$ with $100 \mathrm{~Hz}$ spacing fall inside the $1 \mathrm{kHz}$ resolution bandwidth, increasing the reading of $20 \mathrm{~dB}$ to about $100 \mathrm{~dB} \mu \mathrm{V}$, if we assume, to simplify, that they have all the same amplitude. The noise between the peaks caused mostly by short-range leakage is of the coherent type and contains also some small components located at $50 \mathrm{~Hz}$ odd multiples; the terms sum arithmetically over the new $1 \mathrm{kHz}$ bandwidth giving a $40 \mathrm{~dB}$ increase, that corresponds again to about $100 \mathrm{~dB} \mu \mathrm{V}$. The sum of the two $100 \mathrm{~dB} \mu \mathrm{V}$ terms gives $106 \mathrm{~dB} \mu \mathrm{V}$, that well correspond to those observed in Fig. 4(b). The first switching component at $43 \mathrm{kHz}$ with $100 \mathrm{~Hz}$ RBW in Fig. 4(b) (light grey) has a peak of $60 \mathrm{~dB} \mu \mathrm{V}$, that also well corresponds to the EMI receiver peak of $45 \mathrm{~dB} \mu \mathrm{V}$, observing the $20 \mathrm{~dB}$ increase due to bandwidth and that the narrow modulation of the LB SMPS has components that rapidly fall off along the sides of each peak (so we cannot assume that all peaks falling within the same RBW have the same amplitude). 


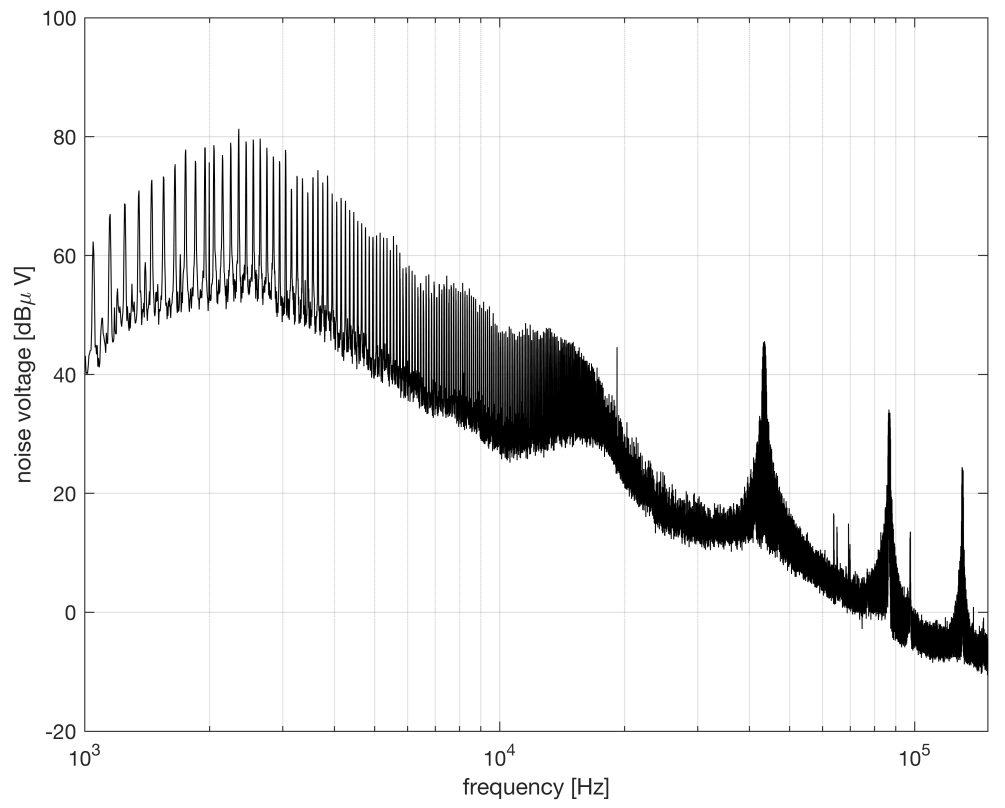

Figure 3: Line spectrum of LB P25 measured with EMI receiver and RBW = $10 \mathrm{~Hz}$. 


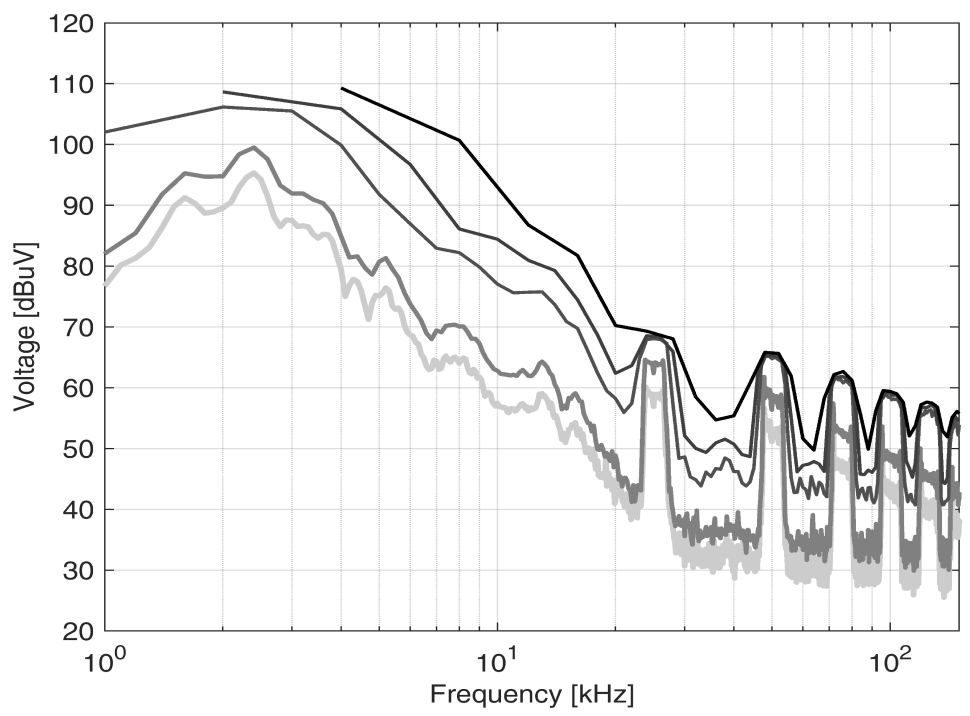

(a)

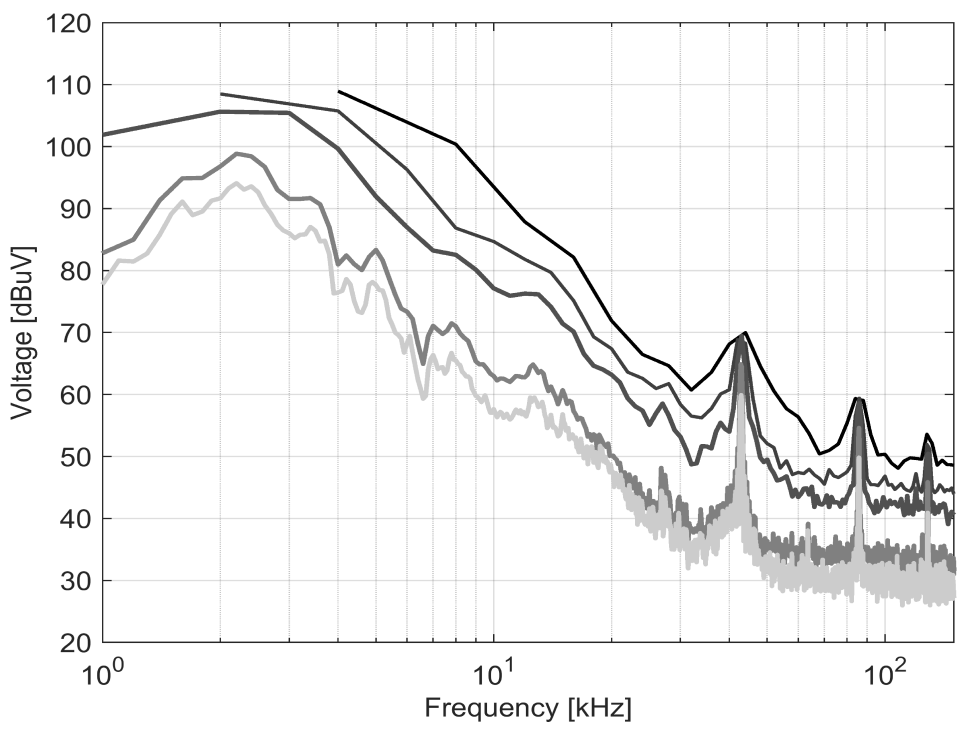

(b)

Figure 4: STFT spectra for (a) KT P25 (waveform in Fig. 2), (b) LB P25, Hann window, $50 \%$ overlap, decreasing time window length $T=10,5,1,0.5,0.25 \mathrm{~ms}$ (from light grey to black curve). 


\subsection{Accuracy, Systematic errors, and Uncertainty}

Calculated spectra are affected by systematic errors caused by window length $T$, due to averaging of signal components and too a narrow frequency resolution, and by weird synchronization with the repetition rate of $100 \mathrm{~Hz}$ (with the term "weird" we indicate that the sequence of windows fixed by the choice of the initial time interval may miscut the signal, so that no window contains all the relevant portions of the signal). Once systematic errors are removed or under control by selection of $T$ and $p$ (the latter is less critical, provided it is not 0 , see end of Sec. 4.3), applying max hold, the remaining variability may be ascribed to the uncertainty of the method. This is statistically analyzed and displayed with

boxplots in sec. 4 where the mentioned max hold value should be retained as the correct estimate of the amplitude. Smoothing windows, excluding Flat-Top, show altogether a spread of maxima of less than $0.5 \mathrm{~dB}$, the Flat-Top always estimating larger amplitudes, anticipating the result of a shorter time window. The spread of maxima due to the overlap, excluding $p=0 \%$ that in some cases gives a significantly lower value, is limited to less than $0.1 \mathrm{~dB}$.

\section{Detailed Results and Quantitative Analysis}

The line spectrum measured with the EMI receiver with low RBW $(10 \mathrm{~Hz})$ (see Fig. 2 as example) is considered the reference for the spectrum details and amplitude estimate.

\subsection{Amplitude accuracy}

The choice of the smoothing window has influence on the accuracy of the amplitude estimate (Fig. 5) and represents a source of systematic error. Starting from a situation where all spectra are corrected for the different coherent gain values of the selected smoothing window, it is possible to observe the peak at the fundamental in Fig. 5(b) that increases going from the Rectangular (in light grey) to the Hann and Gaussian, Hamming and Blackman-Harris, and then Flat-Top. The spread of amplitude values is not only caused by the amount 
of noise floor captured by larger ENBW values, but also by the different slope and sideband attenuation intercepting the adjacent line spectrum components (visible in Figs. 7 and 8). A broader window yields larger estimates, closer to the true value (represented by the top curves in Fig. 7). The Flat-Top, for instance, anticipates the growth of the peaks for increasing RBW, at the expense of a longer window duration by about a factor 2 (the ratio of the ENBW values) to keep the same effective frequency resolution.

The amplitude accuracy attained with the Flat Top window and the other tapering windows (represented by a Hann window) is again emphasized in Figure 6 for a different SMPS (SH P90) operating with a random modulation: even for $200 \mathrm{~Hz}$ RBW the Flat Top correctly estimates the amplitude of the switching components with the green curve almost touching the other two curves at $1 \mathrm{kHz}$ and $2 \mathrm{kHz}$ RBWs; conversely, the amplitude of the nonstationary components caused by the overall switching pulse shape increases with RBW and using the Flat Top, as already pointed out.

\subsection{Frequency resolution}

The effect of the frequency resolution $d f$ is considered with respect to the amount of noise floor, separation of components and resulting amplitude. Small enough $d f$ values (less than the $100 \mathrm{~Hz}$ spacing) give a detailed representation of the underlying line spectrum, as shown in Fig. 8 the line spectrum becomes an envelope spectrum, as soon as $d f$ approaches $100 \mathrm{~Hz}$. The effect of ENBW can be recognized: for the Flat-Top with 3.77 bin a $50 \mathrm{~ms}$ time window is barely sufficient to recognize the line spectrum; the Gaussian window with only 1.45 bin follows well peaks and valleys. The effect of the captured noise floor can be seen in the difference of the estimated peak amplitude for the same $d f$ condition, as reported in the caption of Fig. 8 it is easy to see that the peak amplitude estimates for the largest RBW are very similar and for the analyzed data the error is only $0.3 \mathrm{~dB}$. The $5 \mathrm{~Hz}$ frequency resolution very accurately shows the $100 \mathrm{~Hz}$ spaced spectrum lines at the expense of a heavy unacceptable amplitude underestimate. 


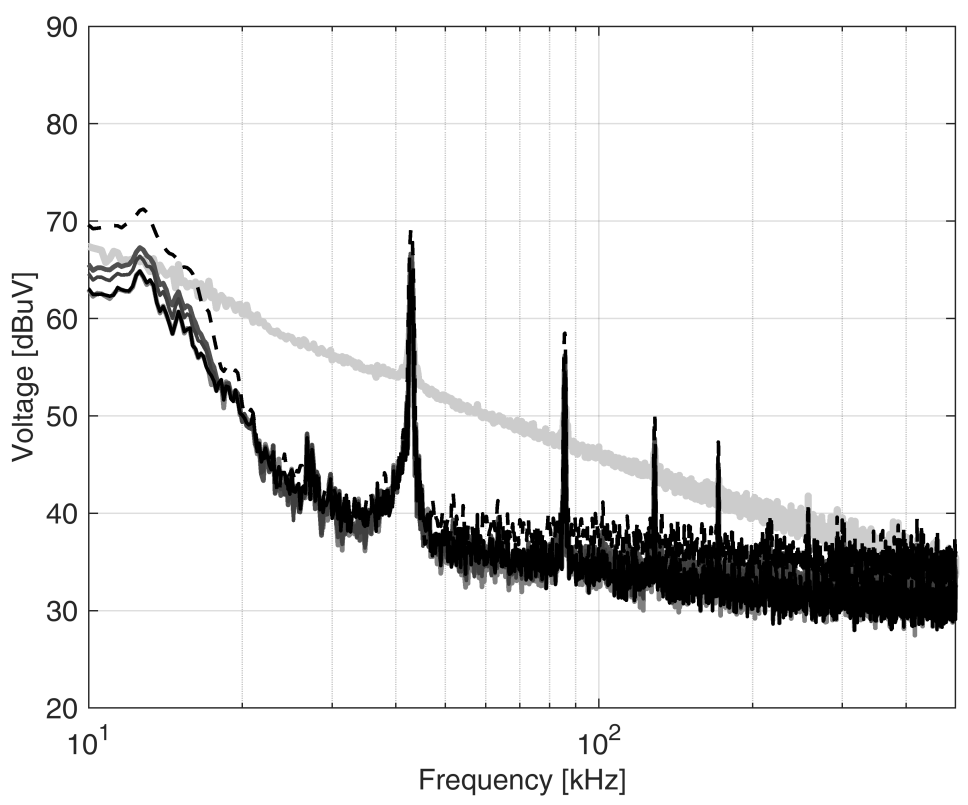

(a)

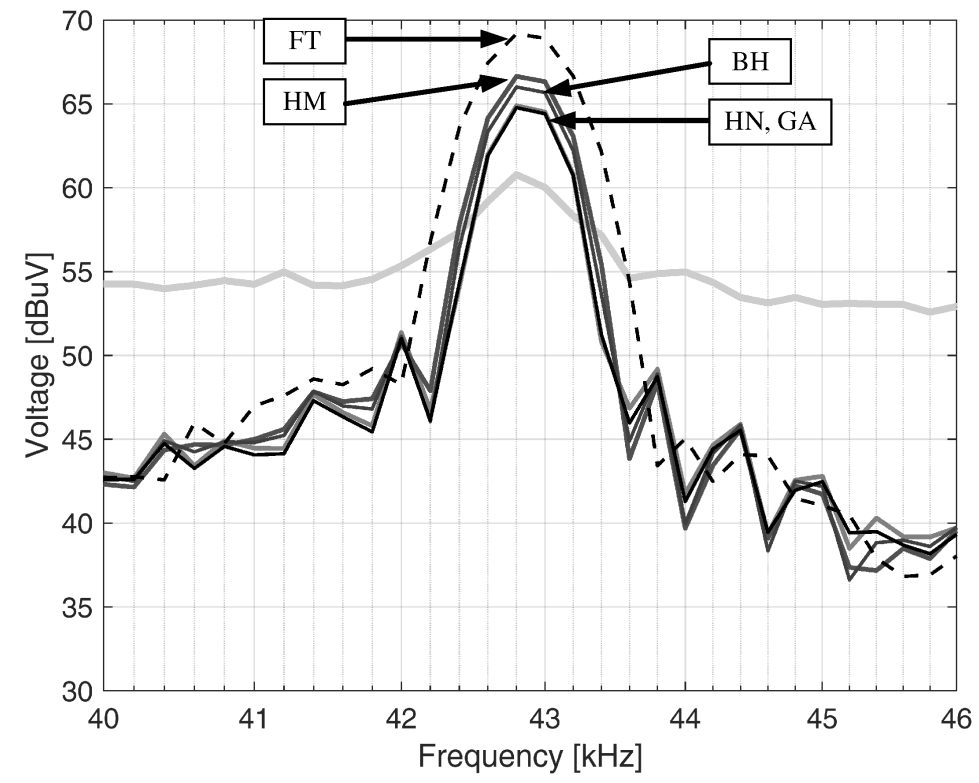

(b)

Figure 5: STFT spectra for LB P25, $200 \mathrm{~Hz}, 50 \%$ overlap and different smoothing windows. 


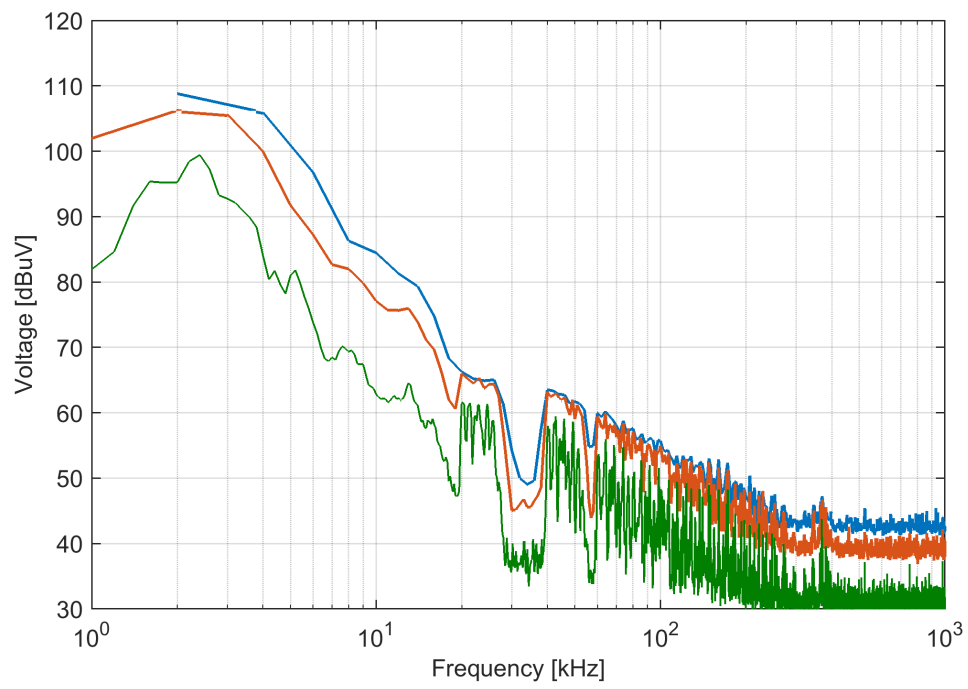

(a)

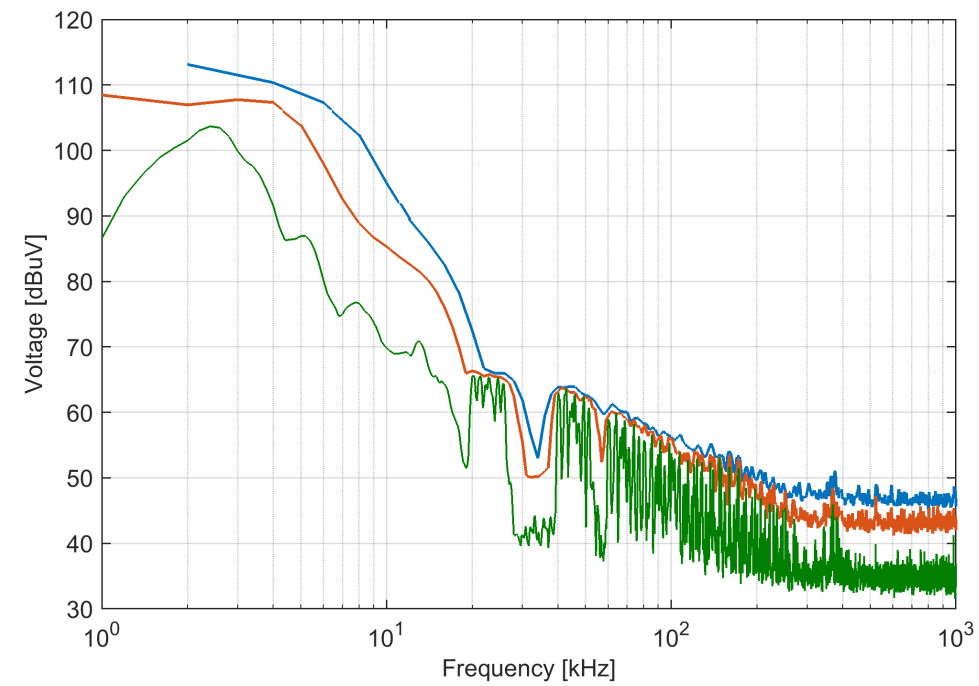

(b)

Figure 6: STFT spectra for SH P90, $50 \%$ overlap, RBW $=2 \mathrm{kHz}, 1 \mathrm{kHz}$ and $200 \mathrm{~Hz}$ for blue, orange ad green curves: (a) Hann and (b) Flat-Top windows. 
Considering that the frequency components highlighted by the smallest $d f$ values are coherent, they must be summed arithmetically when they combine under a larger $d f$ value: thus estimated amplitude grows up almost linearly with RBW, although there is a significant decay of the lateral lines, so that the contribution of those farther from the center line is much less relevant (from which the Flat-Top reaching faster the "saturated" amplitude value for that bin); the linear increase with RBW can thus be held correct for the narrower RBWs for which the bell shaped spectrum lines have a similar height and not a pronounced slope [44].

\subsection{Uncertainty of the amplitude estimate}

Statistical properties of the estimates are first demonstrated by comparison of the curves of the median and the two profiles of the inter-quartile levels ( $25 \%$ and $75 \%$ ), compared to the top curve obtained with the max hold (see Figure 9). Then a more refined graphical representation is implemented by means of boxplots, where the impact of different windows, overlap $p$ and resolution frequency $d f$ on the estimated amplitude of spectral peaks is considered.

The curves in Figure 9 show that the dispersion described by the interquartile profiles is smaller at high frequency (less than $\pm 5 \mathrm{~dB}$ ) and larger for the low frequency components. The max hold profile is much larger, indicating that the distribution at almost all frequency bins is long tailed, except for those frequency intervals around $30-38 \mathrm{kHz}$ and $57 \mathrm{kHz}$, where there are no relevant components of emission.

Calculated spectra are thus subject to a significant dispersion and characterized by systematic errors for some specific parameters combinations. Boxplots allow a better and more complete graphical representation of the statistical characteristics. They show the median (red horizontal bar), the inter-quartile levels (25\% and 75\%, also known as Q1 and Q3, respectively, represented by the upper and lower blue edges of the box) and the upper and lower adjacent values (represented by the black horizontal dashes of the whiskers), which correspond to the largest or smallest data point which is not an outlier (red asterisks). The 


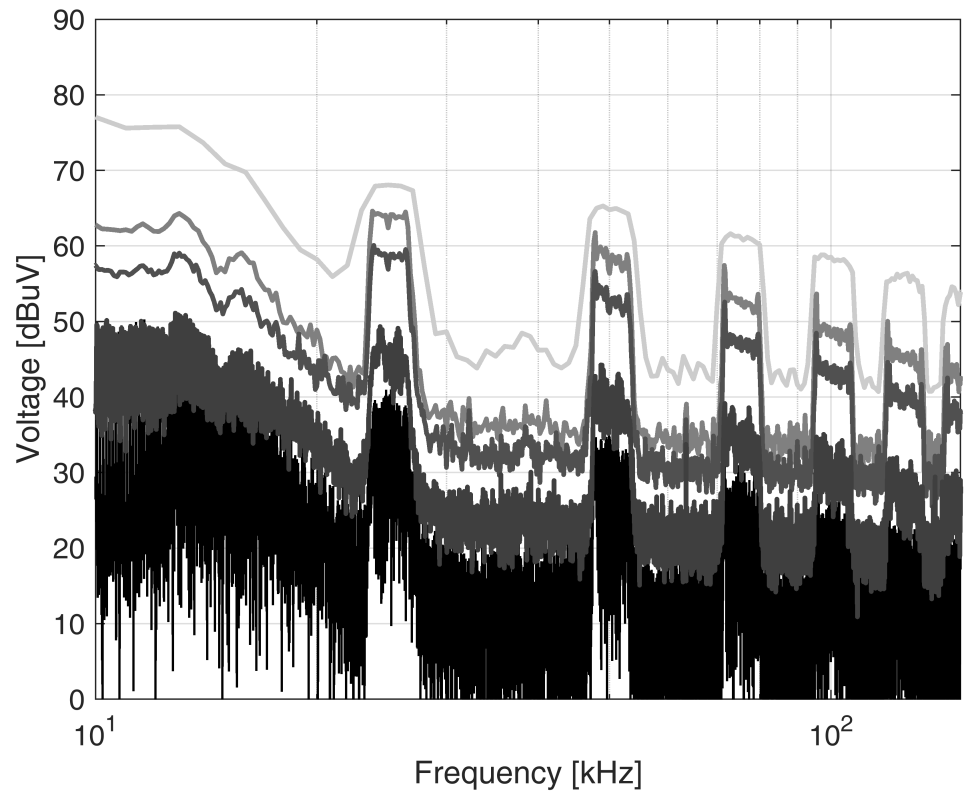

(a)

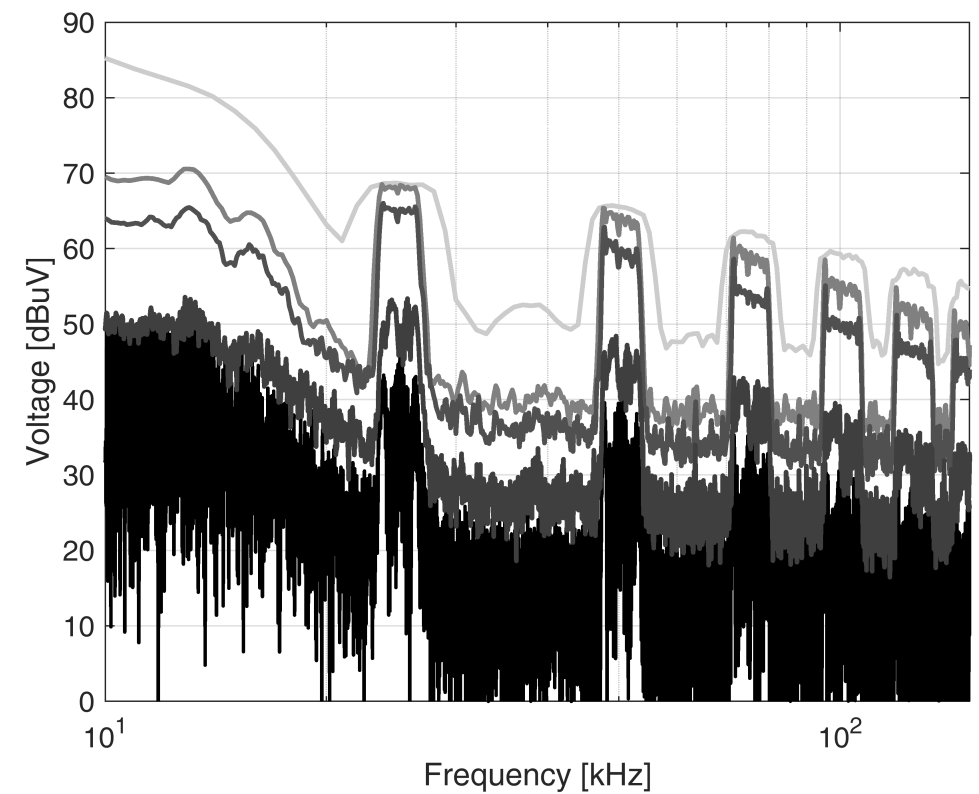

(b)

Figure 7: STFT spectra for KT P25, $50 \%$ overlap, RBW $=1 \mathrm{kHz}, 200 \mathrm{~Hz}$, $100 \mathrm{~Hz}, 20 \mathrm{~Hz}, 5 \mathrm{~Hz}$ for top (light gray) to bottom (black) curves: (a) Hanning and (b) Flat-Top windows. 


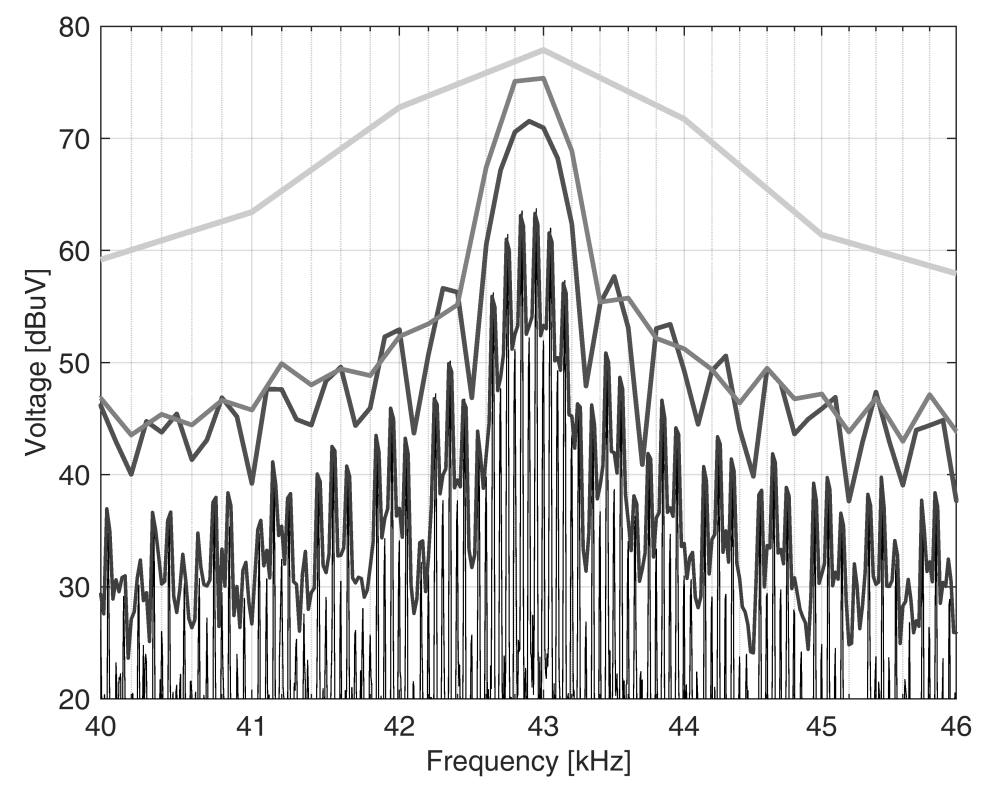

(a)

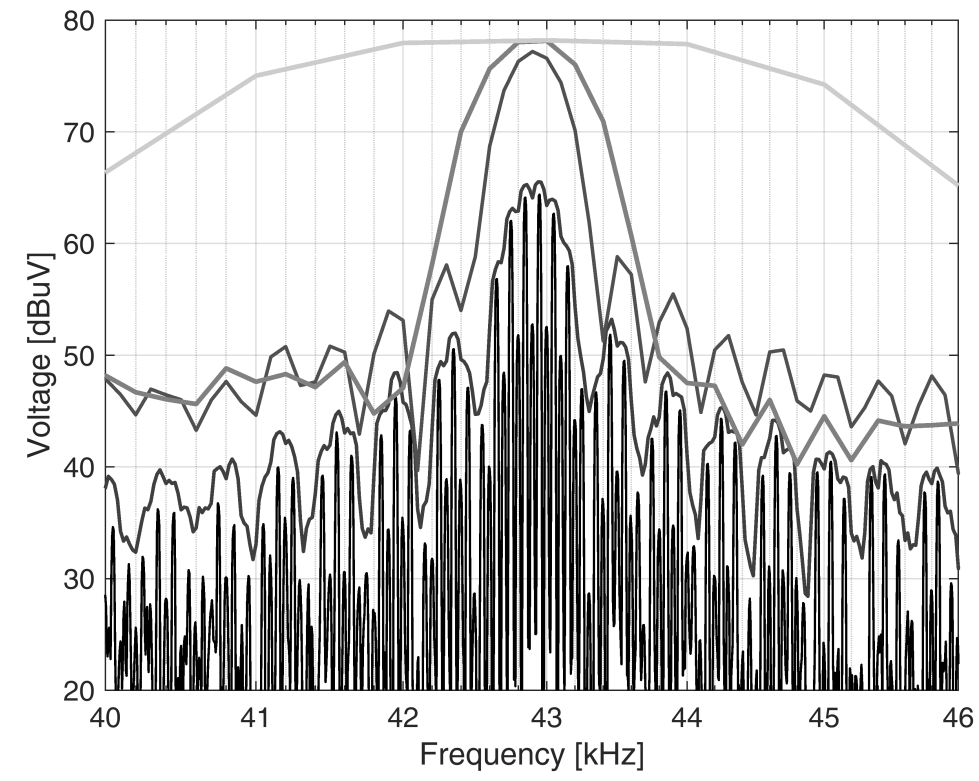

(b)

Figure 8: STFT spectra for LB P90, $50 \%$ overlap, RBW $=1 \mathrm{kHz}, 200 \mathrm{~Hz}$, $100 \mathrm{~Hz}, 20 \mathrm{~Hz}, 5 \mathrm{~Hz}$ for top (light gray) to bottom (black) curves: (a) Gaussian and (b) Flat-Top windows. Peak amplitude at RBW $=1 \mathrm{kHz}, 200 \mathrm{~Hz}$ and $100 \mathrm{~Hz}$ is (a) $77.9,75.4,71.5$ and (b) $78.2,78.1,77.2 \mathrm{~dB} \mu \mathrm{V}$, respectively. 


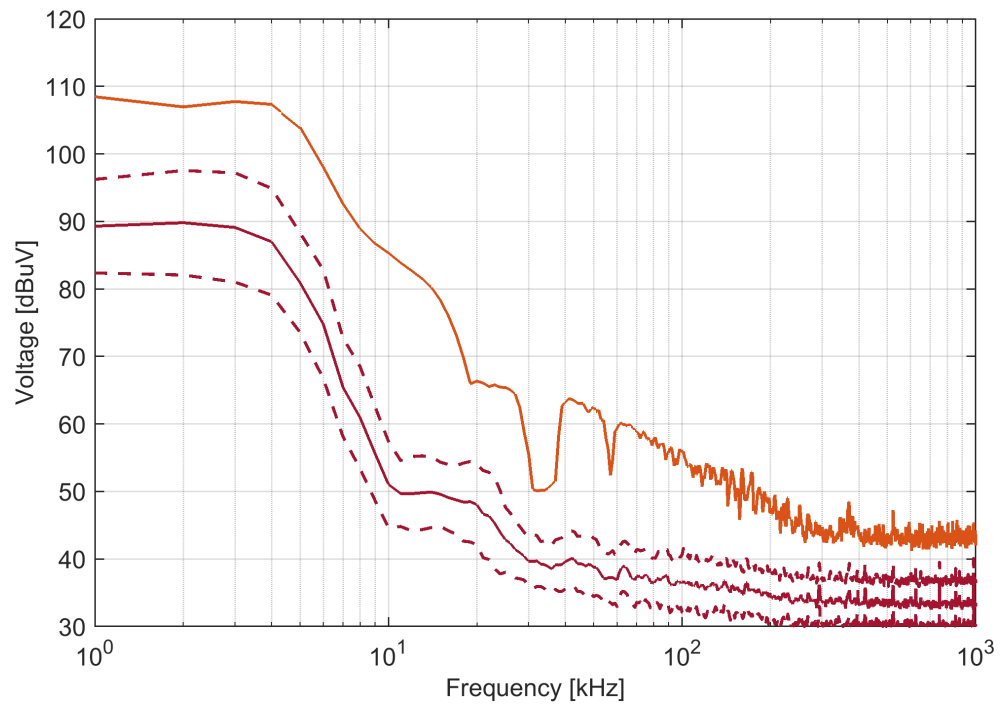

Figure 9: Spectrum of SH P25 measured with $1 \mathrm{kHz}$ frequency resolution: median (purple solid), inter-quartile levels (purple dashed) and max hold (light brown).

use of boxplots aims at a robust statistical characterization. The outliers are the data points below Q1 $-1.5 \times$ IQR or above Q3 $+1.5 \times$ IQR, which are referred as Tukey fences [45, being IQR = Q3 - Q1 the inter-quartile range, i.e., where $50 \%$ of data fall. In case outliers are present, the whiskers represent the largest or smallest data points and not the Min and Max values of the data. The notch in the boxplots displays the confidence interval around the median $m$ which is normally based on $m \pm 1.57 \times \mathrm{IQR} / \sqrt{n}$ [46]. If the notches for two boxplots do not overlap, it can be regarded as strong evidence that their medians differ (i.e. the boxplot distributions are different).

Several spectral estimates have been carried out using a set of $T$ values $S_{T}$ avoiding the identified degenerate cases (so $S_{T}=1,2,2.5,3,5,7.5,15,25 \mathrm{~ms}$ ) and spanning over the most common overlap $p$ values $\left(S_{p}=0 \%, 25 \%, 50 \%, 75 \%\right.$ ). The results of this graphical analysis are shown in Figs. 10 and 11.

Using $T=5 \mathrm{~ms}$, a sub-multiple of the $10 \mathrm{~ms}$ pulse repetition rate, leads to a larger dispersion and several outliers, as shown in Fig. 10 and 11 . The estimated 
amplitude reduces with increasing time window length $T$ due to the unavoidable averaging and compensation of signal portions with opposite polarity). Max hold estimates are similar for $T$ between 2 and $7.5 \mathrm{~ms}$, with the exception of $T=$ $2.5 \mathrm{~ms}$ (again synchronized with $10 \mathrm{~ms}$ ), and slightly smaller than the estimate obtained for $T=1 \mathrm{~ms}$. As it can be noticed from Figs. 10 (a) and (b) and 11 (a) and (b), the notches of the boxplots do not overlap; this means that changing the frequency resolution produces different sets of data with different distributions and therefore the frequency resolution is a key parameter strongly affecting the analysis.

The spread of the amplitude estimate is almost unchanged for all the examined smoothing windows in Fig. 10 and 11. The almost aligned position of the maxima justifies the recommendation of applying the max hold to the calculated STFT. As already observed, the Flat-Top anticipates the estimate thanks to its larger ENBW; the estimate with the Rectangular window suffers - as known - from spectral leakage. As it can be noticed from the plots, the notches of the boxplot relevant to the Rectangular window are not overlapping with the other boxplot notches; this windows seems then to significantly affect the analysis.

The overlap condition $p=0 \%$ (no overlap) leads in some cases to underestimation of the maximum or larger spread, as shown in Fig. 10 and 11 (the evidence was obtained by plotting the same boxplot, but removing the cases for $p=0 \%$ observing that the outliers at the bottom disappeared); moreover, the notches of the boxplot relevant to $p=0 \%$ in some cases do not overlap with the other boxplot notches, thus suggesting a different median of the relevant distribution; using some percentage of overlap is then recommended $(50 \%$ is generally easier to implement).

It is also observed that the largest estimate is obtained with $\mathrm{RBW}=2 \mathrm{kHz}$, in line with the recommendation of the IEC 61000-4-30, but also the amplitude estimates resulting from $\mathrm{RBW}=1 \mathrm{kHz}$ are very close: we may say that much of the difference for the first peak of both LB P25 (Fig. 10(a)) and KT P25 (Fig. 11(a)) is due to the larger collection of the background noise for the $2 \mathrm{kHz}$ 
setting. For the analyzed SMPSs, since the switching burst may last up to about $1 \mathrm{~ms}$, in a few cases the readout with $\mathrm{RBW}=2 \mathrm{kHz}$ was slightly lower than for $1 \mathrm{kHz}$ : this occurred for less than $1 \%$ of all cases and the maximum difference was about $0.2 \mathrm{~dB}$.

As already pointed out, the Flat Top has a larger ENBW and anticipates the estimate compared to the other windows. Considering that the proposed method uses the max hold function applied to the calculated DFT spectra, the comparison of the maxima obtained with the different windows shows that there is a difference of the maxima of $0.9-1.2 \mathrm{~dB}$ for the $\mathrm{LB}$ and $0.5-0.6 \mathrm{~dB}$ for the KT (using spread-spectrum modulation), as appears in Table 3, by comparing the mean values in the two rightmost columns.

In order to complete the analysis, the results for another configuration, P90, of the LB and KT SMPSs and for P25 and P90 for the BA SMPS are shown in Fig. 12. The boxplots present the amplitude of the first peak as a function of frequency resolution. Comparing Fig. 12 with Figs. 10 (a), (b) and Fig. 11 (a), (b) it can again be noticed that the notches of the boxplots do not overlap, yielding strong evidence that the medians of the distributions differ and confirming the importance of frequency resolution in the analysis. Moreover, as previously noticed the $200 \mathrm{~Hz}$ frequency resolution is either responsible of largest dispersion (for LB P90 and KT P90) or highest number of outliers (for BA SMPS). Eventually, observing Fig. 12 (c), (d), besides non overlapping medians one can notice that the maxima of the distributions decrease with decreasing frequency resolutions; in the case of BA SMPS, thus, it seems that $2 \mathrm{kHz}$ is the minimum frequency not to underestimate the first peak.

As soon as all the RBW values and windows are used and the maximum is extracted, the resulting amplitude estimates are all aligned whatever the overlap percentage, although we have already noticed that $0 \%$ is exposed to larger dispersion, outliers and in principle the risk of underestimating some signal components based on the following mechanism, already discussed in sec. 3.1 the combination of a time window length $T$ equal to the repetition period of the pulses, combined with a no overlap setting $(p=0 \%)$, with a synchronized acqui- 
Table 3: Mean and dispersion for max hold estimates of LB and KT SMPSs first and second peak of emission vs. overlap and window

\begin{tabular}{|c|c|c|c|c|}
\hline SMPS peak & overlap & $\begin{array}{c}\text { window } \\
\text { (excl. R) }\end{array}$ & $\begin{array}{c}\text { window } \\
\text { (excl. R \& FT) }\end{array}$ & $\begin{array}{c}\text { window } \\
\text { (only FT) }\end{array}$ \\
\hline \hline LB P25 pk1 & $70.767 / 0$ & $69.756 / 0.503$ & $69.554 / 0.097$ & 70.767 \\
\hline LB P25 pk2 & $60.425 / 0.286$ & $59.829 / 0.382$ & $59.678 / 0.104$ & 60.586 \\
\hline KT P25 pk1 & $68.720 / 0.101$ & $68.362 / 0.299$ & $68.260 / 0.185$ & 68.870 \\
\hline KT P25 pk2 & $65.983 / 0.0008$ & $65.538 / 0.235$ & $65.449 / 0.096$ & 65.984 \\
\hline
\end{tabular}

sition e.g. caused by the DSO trigger that position the peak at the beginning of the recording, will always underestimate the amplitude and never recover from it all along the recording, in particular if smoothing windows are used, that attenuate the signal at the two ends. This may also occur for sub-multiples of $10 \mathrm{~ms}$, such as $5 \mathrm{~ms}$.

In general spread-spectrum modulation might show some inconsistencies in the results, such as: lack of a clear monotonic increase of amplitude with increasing RBW, especially for high-frequency components, as observed above for the comparison of $1 \mathrm{kHz}$ and $2 \mathrm{kHz}$ RBW values; uncommon statistical distribution of amplitude values, resulting in a larger number of outliers, as detected by the boxplot routine.

\section{Conclusion}

The time-domain measurement of conducted emissions of Switched-Mode Power Supplies (SMPSs) has been considered compared to the frequency domain method, analyzing the effects of the parameters of the Short-Time Fourier Transform (STFT), namely time window length $T$ and resulting frequency resolution $d f$, the overlap $p$, and the smoothing windows. The analysis is based on theoretical assumptions verified by experimental results on a set of six different SMPSs. 


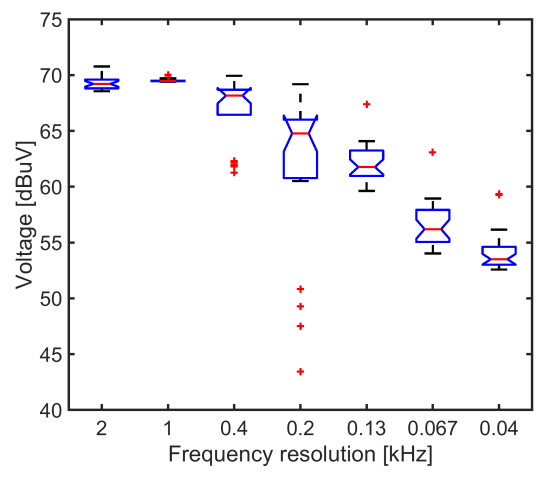

(a)

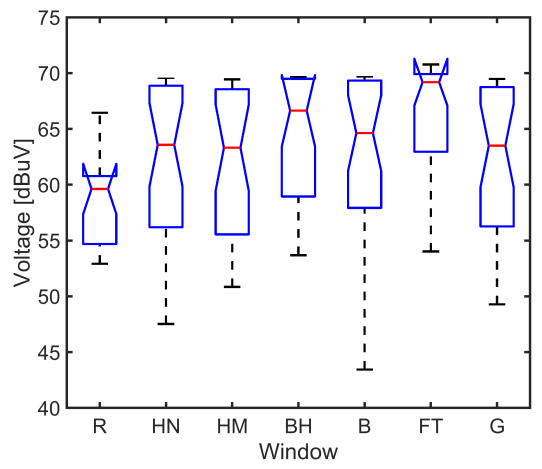

(c)

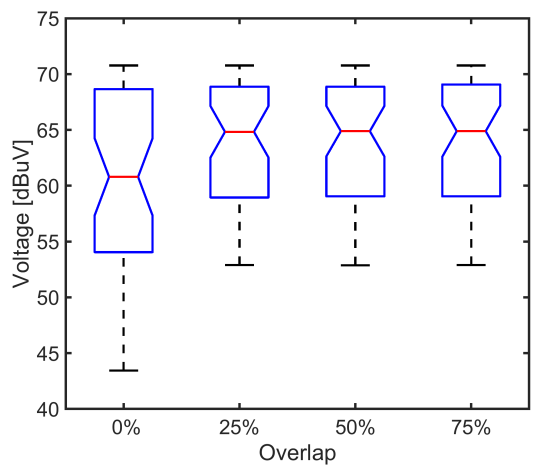

(e)

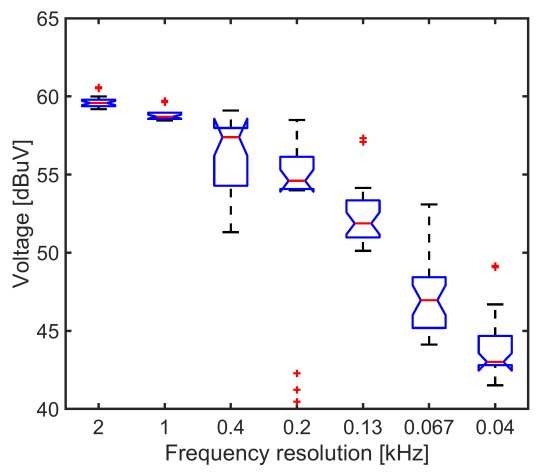

(b)

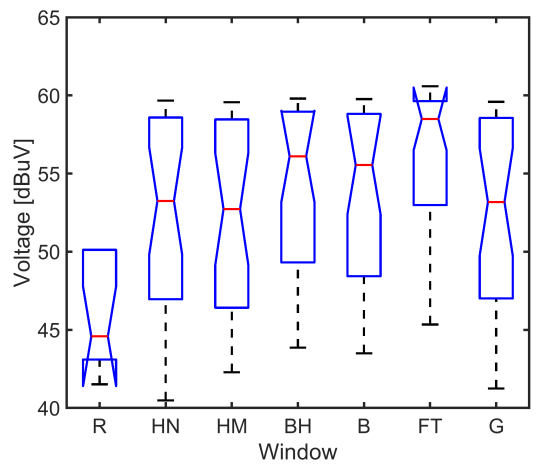

(d)

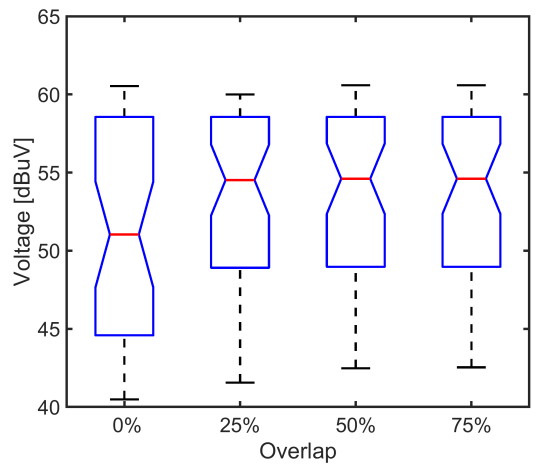

(f)

Figure 10: Boxplot of the amplitude of the first (a, c, e) and second peak (b, $\mathrm{d}, \mathrm{f})$ for LB P25, as a function of $(\mathrm{a}, \mathrm{b})$ frequency resolution (corresponding to the $T$ values in $\left.S_{T}\right)$, (c, d) smoothing window, $(\mathrm{e}, \mathrm{f})$ overlap percentage $p$ for values in $S_{p}$. 


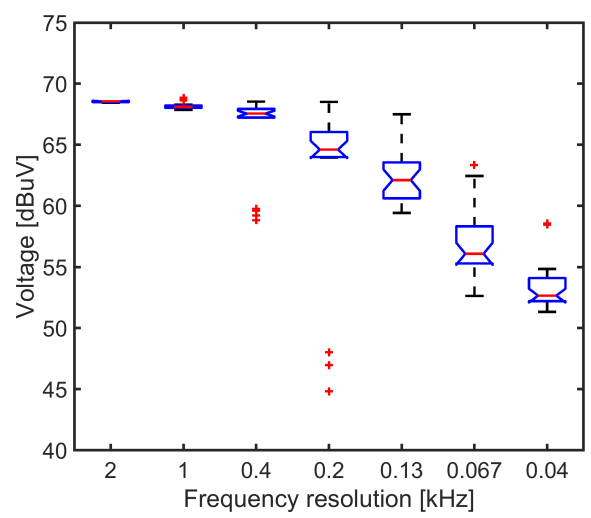

(a)

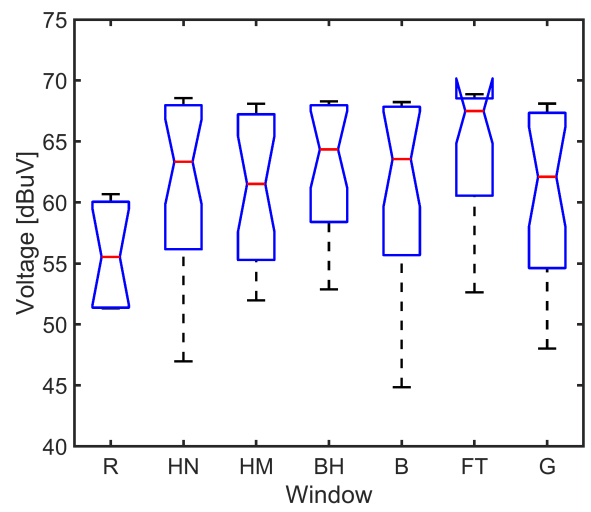

(c)

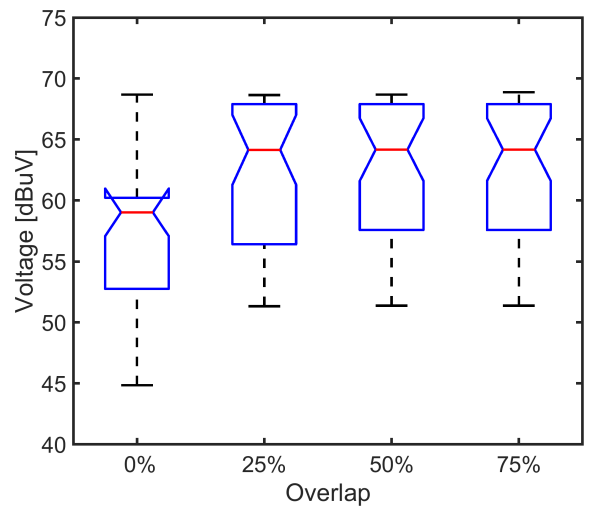

(e)

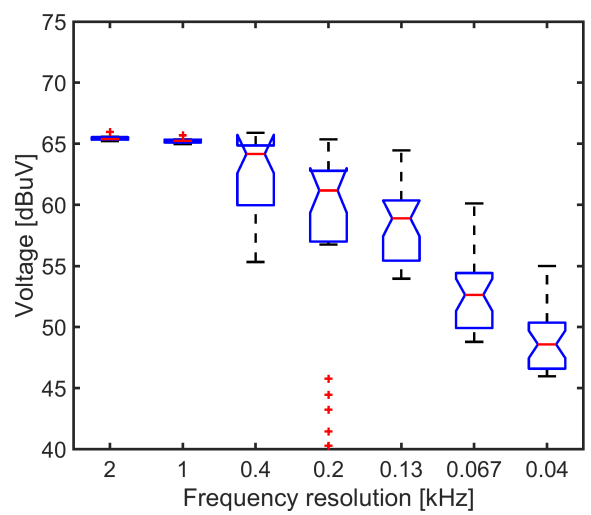

(b)

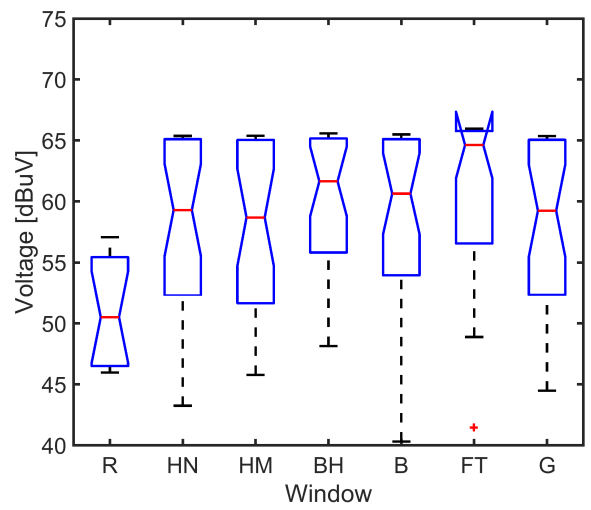

(d)

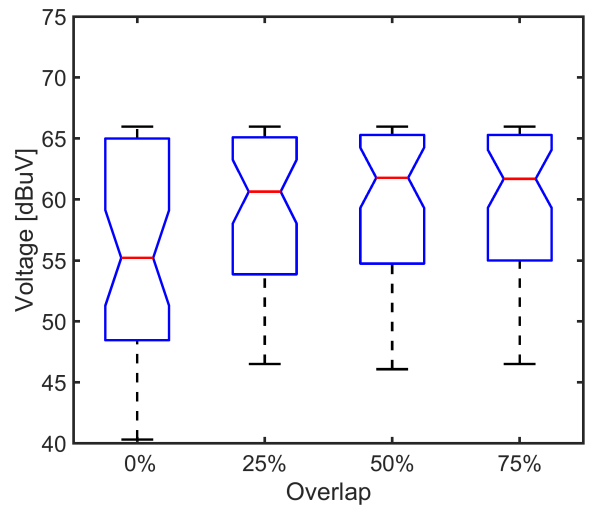

(f)

30

Figure 11: Boxplot of the amplitude of the first (a, c, e) and second peak (b, $\mathrm{d}, \mathrm{f})$ for KT P25, as a function of $(\mathrm{a}, \mathrm{b})$ frequency resolution (corresponding to the $T$ values in $\left.S_{T}\right),(\mathrm{c}, \mathrm{d})$ smoothing window, $(\mathrm{e}, \mathrm{f})$ overlap percentage $p$ for values in $S_{p}$. 


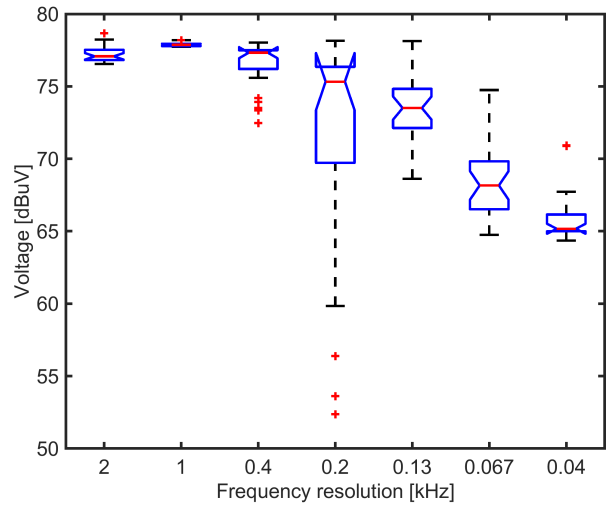

(a)

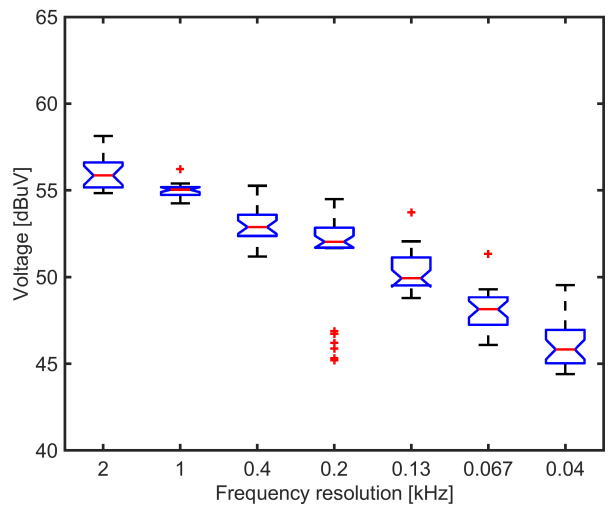

(c)

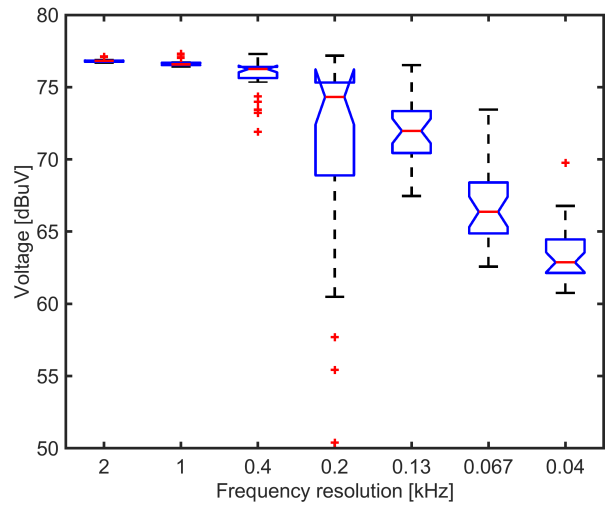

(b)

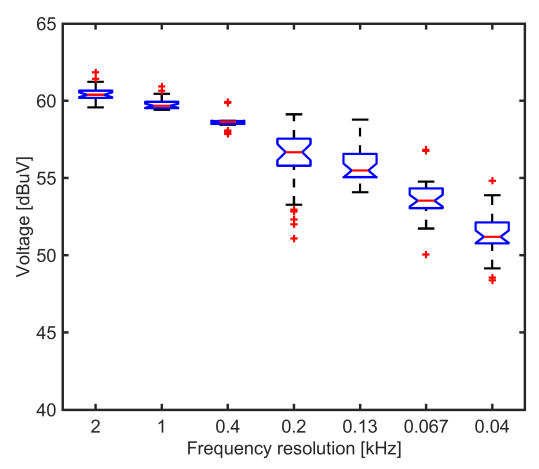

(d)

Figure 12: Boxplot of the amplitude of the first peak for (a) LB P90, (b) KT P90, $(\mathrm{c}, \mathrm{d})$ BA P25 and P90, as a function of frequency resolution, complementing the previous boxplot analysis. 
Systematic errors have been identified for the estimate of the amplitude of spectral components related to undesirable synchronization with the pulse repetition rate (i.e. $T=5,10$ and $20 \mathrm{~ms}$ ) and extreme values of the overlap percentage (i.e. $p=0$, no overlap). The behavior of the spectral estimates for variable frequency resolution (corresponding to the RBW of the frequency domain measurements) has also been evaluated, considering the coherent behavior of the various spectral lines and leakage components in between. It was also observed that frequency resolution influences the distribution of the amplitude values, as pointed out by commenting the shape of the boxplot results.

In general, broad tapering windows are preferable (such as the Flat-Top, although the other examined windows give also repeatable results) and/or the use of a short enough window (e.g. $T=0.5-1.5 \mathrm{~ms}$ ) to slice the signal accurately, followed by max hold applied to the absolute value of the spectral components. Of course, the max-hold setting necessary for an accurate estimate of the spectral amplitude reduces the accuracy of tracking vs. time and does not allow to preserve phase information.

The accuracy of the frequency estimate of components is not a critical aspect, although broader smoothing windows tend to worsen it; interpolation can recover easily the location of signal components lying between frequency bins with a more than satisfactory accuracy. For amplitude interpolation has not been considered in this work, since the focus was on the variability of spectrum data to which various interpolation methods can be applied at a later stage.

Once max hold and a conveniently large resolution frequency $(1-2 \mathrm{kHz})$ are chosen, the remaining uncertainty of the amplitude estimate is quite small, showing a spread of $0-0.3 \mathrm{~dB}$ with respect to the overlap values and $0.9-$ $1.2 \mathrm{~dB}$ with respect to the smoothing windows excluding the Flat Top; using the Flat Top, as advised, removes the uncertainty related to the used window, maximizing the amplitude estimate.

\section{References}




\section{References}

[1] D. Thomas, Conducted emissions in distribution systems (1 kHz-1 MHz), IEEE Electromagnetic Compatibility Magazine 2 (2) (2013) 101 - 104.1

[2] T. J. Densem, P. S. Bodger, J. Arrillaga, Three phase transmisssion system modelling for harmonic penetration studies, IEEE Transactions on Power Apparatus and Systems PAS-103 (2) (1984) 310-317. 1

[3] P. F. Ribeiro, L. C. Fonseca, Considerations on the accuracy of three-phase harmonic penetration studies in transmission systems: A case study, in: Proc. ICHPS V International Conference on Harmonics in Power Systems, Atlanta, GA, USA, 1992, pp. 57-64. 1

[4] E. A. Larsson, M. H. Bollen, M. G. Wahlberg, C. M. Lundmark, S. K. Ronnberg, Measurements of high-frequency $(2-150 \mathrm{kHz})$ distortion in lowvoltage networks, IEEE Transactions on Power Delivery 25 (3) (2010) 1749 - 1757. 1

[5] D. Frey, J. Schanen, S. Quintana, M. Bollen, C. Conrath, Study of high frequency harmonics propagation in industrial networks, in: Proc. IEEE International Symposium on Electromagnetic Compatibility, Rome, Italy, 2012, pp. $1-5$.

[6] G. F. Bartak, A. Abart, EMI of emissions in the frequency range $2 \mathrm{kHz}$ - $150 \mathrm{kHz}$, in: Proc. CIRED 22nd International Conference on Electricity Distribution, Stockholm, Sweden, 2013, pp. 1-4.

[7] M. Klatt, J. Meyer, P. Schegner, Comparison of measurement methods for the frequency range of $2 \mathrm{kHz}$ to $150 \mathrm{kHz}$, in: Proc. International Conference on Harmonics and Quality of Power, ICHQP, Bucharest, Romania, 2014, pp. $818-822$. 1

[8] L. Sandrolini, D. W. P. Thomas, M. Sumner, C. Rose, Measurement and evaluation of the conducted emissions of a DC/DC power converter in the 
frequency range 2-150 kHz, in: Proc. 2018 IEEE Symposium on Electromagnetic Compatibility, Signal Integrity and Power Integrity (EMC SI\&PI), Long Beach, CA, USA, 2018, pp. 345-350.

[9] A. Mariscotti, L. Sandrolini, Techniques for the analysis of time-domain conducted emissions of SMPS in smart grids, in: Proc. 2019 IEEE International Conference on Environment and Electrical Engineering and 2019 IEEE Industrial and Commercial Power Systems Europe (EEEIC / I\&CPS Europe), Genoa, Italy, 2019, pp. 1-6.

[10] L. Ensini, L. Sandrolini, D. W. P. Thomas, M. Sumner, C. Rose, Conducted emissions on DC power grids, in: Proc. 2018 International Symposium on Electromagnetic Compatibility (EMC EUROPE), Amsterdam, the Netherlands, 2018, pp. 214-219.

[11] M. H. Hedayati, V. John, Filter configuration and PWM method for singlephase inverters with reduced conducted EMI noise, IEEE Transactions on Industry Applications 51 (4) (2015) 3236-3243.

[12] S. Singh, S. B., G. Bhuvaneswari, V. Bist, A power quality improved bridgeless converter based computer power supply, in: Proc. 2014 6th IEEE Power India International Conference (PIICON), Delhi, India, 2014, pp. 1-6. 1

[13] CISPR 14-1, Electromagnetic compatibility - requirements for household appliances, electric tools and similar apparatus part 1: Emission, CISPR (2016). 12.2

[14] CISPR 15, Limits and methods of measurement of radio disturbance characteristics of electrical lighting and similar equipment, CISPR (2018).

[15] EN 50065-1, Signalling on low-voltage electrical installations in the frequency range $3 \mathrm{kHz}$ to $148,5 \mathrm{kHz}$ part 1: General requirements, frequency bands and electromagnetic disturbances, CENELEC (2012).

[16] IEC 61000-4-19, Electromagnetic compatibility (EMC) - part 4-19: Testing and measurement techniques - test for immunity to conducted, differential 
mode disturbances and signalling in the frequency range $2 \mathrm{kHz}$ to $150 \mathrm{kHz}$ at a.c. power ports, IEC (2014). 1.2.2

[17] M. Bollen, H. H. Shyar, S. Rönnberg, Spread of high frequency current emission, in: Proc. 22nd International Conference and Exhibition on Electricity Distribution (CIRED 2013), Stockholm, Sweden, 2013, pp. 1-4. 1

[18] S. K. Rönnberg, A. G. Castro, M. H. J. Bollen, A. Moreno-Munoz, E. Romero-Cadaval, Supraharmonics from power electronics converters, in: Proc. 2015 9th International Conference on Compatibility and Power Electronics (CPE), Costa da Caparica, Portugal, 2015, pp. 539-544. doi: 10.1109/CPE.2015.7231133

[19] R. Torquato, W. Xu, W. Freitas, J. Lepka, R. Pascal, Propagation characteristics of high-frequency harmonics in distribution systems, in: Proc. 2016 IEEE Power and Energy Society General Meeting (PESGM), Boston, MA, USA, 2016, pp. 1-5. doi:10.1109/PESGM. 2016.7741220. 1

[20] IEC 61000-4-30, Electromagnetic compatibility (EMC) - part 4-30: Testing and measurement techniques - power quality measurement methods, IEC (2015). $1,3.1$

[21] IEC 61000-4-7, Electromagnetic compatibility (EMC) - part 4-7: Testing and measurement techniques - general guide on harmonics and interharmonics measurements and instrumentation, for power supply systems and equipment connected thereto, IEC (2002). 1, $2.2,2.3$

[22] CISPR-16-1-1, Specification for radio disturbance and immunity measuring apparatus and methods - part 1-1: Radio disturbance and immunity measuring apparatus - measuring apparatus, CISPR (2019). 1

[23] J. Medler, C. Reimer, Timing considerations using FFT-based measuring receivers for EMI compliance measurements, in: Proc. 2014 International Symposium on Electromagnetic Compatibility, Tokyo, Tokyo, Japan, 2014, pp. 93-96. 1 
[24] M. Keller, Comparison of time domain scans and stepped frequency scans in EMI test receivers, White Paper, Rohde\&Schwarz (2014). 1

[25] L. Alfieri, A. Bracale, A. Larsson, New power quality indices for the assessment of waveform distortions from 0 to $150 \mathrm{kHz}$ in power systems with renewable generation and modern non-linear loads, Energies 10 (10) (2017) 1633. doi:10.3390/en10101633. 1

[26] M. Bollen, M. Olofsson, A. Larsson, S. Ronnberg, M. Lundmark, Standards for supraharmonics (2 to $150 \mathrm{khz}$ ), IEEE Electromagnetic Compatibility Magazine 3 (1) (2014) 114 - 119, current distortion;Frequency ranges;Harmonic emissions;Individual devices;International standards;Standard frameworks;Voltage fluctuations;.

URL http://dx.doi.org/10.1109/MEMC.2014.6798813 1

[27] S. Braun, T. Donauer, P. Russer, A real-time time-domain EMI measurement system for full-compliance measurements according to CISPR 16-1-1, IEEE Transactions on Electromagnetic Compatibility 50 (2) (2008) 259267. doi:10.1109/temc.2008.918980, 1

[28] G. Costa, M. Pous, A. Atienza, F. Silva, Time-domain electromagnetic interference measurement system for intermittent disturbances, in: Proc. 2014 International Symposium on Electromagnetic Compatibility, Gothenburg, Sweden, 2014, pp. 833-837. doi:10.1109/EMCEurope.2014. 6931019

[29] M. A. Azpurua, M. Pous, F. Silva, A measurement system for radiated transient electromagnetic interference based on general purpose instruments, in: Proc. IEEE International Symposium on Electromagnetic Compatibility (EMC), 2015. 1

[30] M. Parvis, G. Perrone, A. Vallan, A precompliance EMC test-set based on a sampling oscilloscope, IEEE Transactions on Instrumentation and Measurement 52 (4) (2003) 1220-1223. doi:10.1109/TIM. 1 
[31] J. B. Noshahr, B. M. Kalasar, Evaluating emission and immunity of harmonics in the frequency range of $2-150 \mathrm{kHz}$ caused by switching of static convertor in solar power plants, CIRED - Open Access Proceedings Journal 2017 (1) (2017) 625-628. 1

[32] T. M. Mendes, C. A. Duque, L. R. M. da Silva], D. D. Ferreira, J. Meyer, P. F. Ribeiro, Comparative analysis of the measurement methods for the supraharmonic range, International Journal of Electrical Power \& Energy Systems 118 (2020) 105801. doi:https://doi.org/10.1016/j.ijepes. 2019.105801. 1]

[33] V. Khokhlov, J. Meyer, A. Grevener, T. Busatto, S. Rönnberg, Comparison of measurement methods for the frequency range $2-150 \mathrm{kHz}$ (supraharmonics) based on the present standards framework, IEEE Access 8 (2020) 77618-77630. 1

[34] S. Lodetti, J. Bruna, J. J. Melero, V. Khokhlov, J. Meyer, A robust waveletbased hybrid method for the simultaneous measurement of harmonic and supraharmonic distortion, IEEE Transactions on Instrumentation and Measurement (2020) 1-1doi:10.1109/tim.2020.2981987. 1

[35] A. Mariscotti, Discussion of power quality metrics suitable for DC power distribution and smart grids, in: Proc. 24th IMEKO TC4 Int. Symp., Xi'an, China, 2019.

[36] L. Sandrolini, A. Mariscotti, Signal transformations for analysis of supraharmonic EMI caused by switched-mode power supplies, Electronics 9 (12) (2020) 2088. doi:10.3390/electronics9122088. 1

[37] F. J. Harris, On the use of windows for harmonic analysis with the discrete fourier transform, Proceedings of the IEEE 66 (1) (1978) 51-83. 2.1.2.2

[38] R. Lyons, Understanding Digital Signal Processing, 3rd Edition, Prentice Hall, 2011. 2.1 
[39] B. G. Quinn, Estimation of frequency, amplitude, and phase from the DFT of a time series, IEEE Transactions on Signal Processing 45 (3) (1997) 814-817. 2.1

[40] E. Jacobsen, On local interpolation of dft outputs (1994) [cited May 2019]. URL http://www.ericjacobsen.org/FTinterp.pdf

[41] M. Gasior, J. Gonzalez, Improving FFT frequency measurement resolution by parabolic and gaussian interpolation, AB- Note-2004-021 BDI, CERNAB Div., Geneva, Switzerland (Feb. 2004).

[42] D. Agrez, Weighted multipoint interpolated DFT to improve amplitude estimation of multifrequency signal, IEEE Transactions on Instrumentation and Measurement 51 (2) (2002) 287-292. 2.1

[43] A. J. Collin, A. D. Femine, C. Landi, R. Langella, M. Luiso, A. Testa, The role of supply conditions on the measurement of high-frequency emissions, IEEE Transactions on Instrumentation and Measurement 69 (9) (2020) 6667-6676. doi:10.1109/tim.2020.2992824, 2.3

[44] S. Linkwitz, A. Wilcox, Techniques for measuring narrowband and broadband emi signals using spectrum analyzers, Tech. rep., Hewlett-Packard RF \& Microwave Measurement Symposium and Exhibition 1984 (1984). 4.2

[45] J. W. Tukey, Exploratory Data Analysis, Addison-Wesley Publishing Company, 1977. 4.3

[46] J. M. Chambers, W. Cleveland, B. Kleiner, P. Tukey, Graphical Methods for Data Analysis, CRC Press CRC Press, Boca Raton, FL, USA, 1983. 4.3 